\title{
CARACTERIZACKO DOS PARES DE PONTOS CRITICOS ASSOCIADOS A $F$ E A SUA DERIVADA RADIAL
}

SONNIA REGINA LEITE GARCIA

\author{
DISSERTAÇÃO APRESENTADA AO \\ INSTITUTO DE MATEMATTICA E ESTATISTICA \\ DA \\ UNIVERSIDADE DE SÃO PAULO \\ PARA OBTENÇÃO DO GRAU DE MESTRE EM \\ MATEMATI CA APLICADA
}

AREA DE CONCENTRAÇÃO: MATEMATICA APLICADA ORIENTADOR: Prof. Dr. ANGELO BARONE NETTO

- São Paulo, Agos to de 1985 - 
Ao mestre e amigo

Märio Barone Jünion 
A pretensão de que este trabalho josse individual, isolado, $\bar{e}$ algo, pelo menos absurdo, assim:

Ao Instituto de Matemätica e Estatistica e em especial ao Departa mento de Matemätica Aplicada pela confiança em mim depositada.

Ao Projessor Doutor Ivan de Queiroz Barros, meu pai em termos de formação matemática.

Ao Projessor Doutor Märio Barone Ir., por tudo o que representou sua orientação segura na fase essencial de formação, tanto na graduação como no início do mestrado.

Ao Professor Doutor Mauro de oliveira César e ao Professor Assistente Manuel V. P. Garcia, pelo incentivo e sugestões tanto no desenvolvimento como na redação desta dissertação.

Ao grupo de Sistemas Dinâmicas do I.M.E., pelas palpites e conselhos oportunas.

Ao Professor Doutor Angelo Barone Netto, muito mais que un orientador, a mola desta dissertação, dada a paciência evidenciada nestes ültimos dois anos.

Aos colegas e funcionärios do I.M.E., pela boa vontade sempre rei terada.

Ao Raul pela mágica feita na datilografia deste trabalho, e em tempo record.

A todos os que contribuiram de algum modo neste trabalho.

Muito Obrigado

SÔNIA REGINA LEITE GARCIA

(*) A AUTORA AGRADECE AO CNPq DE QUEM RECEBEU AUXILIO FINANCEIRO DURAN TE OS DOIS PRIMETROS ANOS DE MESTRADO. 
This work has four chapters. The first of them is here by didactic and historic purposes.

Inspired by [3], the purpose of first chapter is to show a demonstration of Sard's Algebraic Theorem without using Tarski-Seidenberg theorem, moreover we make a geome--: trical description of following varietes using that result

$$
\begin{aligned}
& V(f)=\left\{x \in \mathbb{R}^{n} \mid \operatorname{grad} f(x) \text { is radial }\right\} \\
& V^{\perp}(f)=\left\{x \in \mathbb{R}^{n}|<\operatorname{grad} f(x)| x>=0\right\}
\end{aligned}
$$

where $f$ is a polinomial of $n$ variables.

In chapter II we relate the bahavior of a germ $f:\left(\mathbb{R}^{\mathrm{n}} ; 0\right) \stackrel{\mathrm{C}^{1}}{\longrightarrow}(\mathbb{R} ; 0)$ with the behavior or "radial deriva te" of $f, f^{\prime} ;\left(\mathbb{R}^{\mathrm{n}} ; 0\right) \longrightarrow(\mathbb{R} ; 0)$ defined by $f^{\prime}(x)=\langle\operatorname{grad} f(x) \mid x\rangle$ [here $\langle\mid\rangle$ is the usual inner product of $\mathbb{R}^{\mathrm{n}}$ ].

In chapter III we generalize the result of chapter II, considering a generic inner product of $\mathbb{R}^{\mathrm{n}}$.

The Last chapter is an application to Liapunov's stabi lity of Hamiltonian systems of two degree of freedom. 
INTRODUÇAO

CAPITULO I

I-1 Introdução.

I-2 As variedades algébricas $V(f)$ e $V^{\perp}(f)$.

I-3 Decomposição de variedades algébricas.

I-4 Algumas propriedades de $V(f)$ e de $V^{\perp}(f)$.

CAPITULO II

II - I Introdução

II-2 A derivada radial.

II-3 Propriedades de $f^{\prime}$.

II-4 Classificação dos pares $\left(f, f^{\prime}\right)$ para $f:\left(\mathbb{R}^{n} ; 0\right) \stackrel{C^{1}}{\longrightarrow}(\mathbb{R} ; 0)$.

II-5 Uma classe em que os pares ( $\left.f, f^{\prime}\right)$ são triviais.

I -6 Estudo de alguns exemplos.

CAPITULO II I

III-1 Introdução.

II I-2 A derivada "radial" f́.

II -3 Exemplos.

II - 4 Propriedades de $f_{A}^{\prime}$.

III-5 Classificação dos pares ( $\left.f, f_{A}^{\prime}\right)$.

\section{CAPITULO IV}

IV-1 Introdução.

IV-2 Teorema de Liapunov para Instabilidade.

IV-3 Comentários . 
$\begin{array}{lllllllllll}\text { I } & N & T & R & O & D & U & C & \AA & 0\end{array}$ 
Este trabalho compõe-se de quatro capítulos, dos quais o primeiro acha-se aqui por motivos históricos e didáticos.

Motivado pelos resultados apresentados em [3], o primeiro capítulo tem como objetivo apresentar uma demonstra-ção do Teorema Algébrico de Sard sem lançar mão do Teorema de Tarski-Seidenberg, e usar o resultado para descrever geo metricamente as variedades algébricas

$$
\begin{aligned}
& V(f)=\left\{x \in \mathbb{R}^{\mathrm{n}} \mid \operatorname{gradf}(x) \text { é } \operatorname{radia} 1\right\} \\
& \left.\mathrm{V}^{\perp}(f)=\left\{x \in \mathbb{R}^{\mathrm{n}}|<\operatorname{grad} f(x)| x\right\rangle=0\right\}
\end{aligned}
$$

onde $f$ è um polinômio em $n$ variáveis.

Os resultados apresentados no $\S I-3$ versam sobre Estratificação de variedades algébricas, e estão todos contidos em [5] e [6]. Foram apresentados aqui apenas com o intuito de tornar o texto, na medida do possível, auto-suficiente.

No capitulo II, relacionamos o comportamento de um ge me $f:\left(\mathbb{R}^{\mathrm{n}} ; 0\right) \stackrel{\mathrm{C}^{1}}{\longrightarrow}(\mathbb{R} ; 0)$ com o comportamento do germe $f^{\prime}:\left(\mathbb{R}^{n} ; 0\right) \stackrel{C^{O}}{\longrightarrow}(\mathbb{R} ; 0)$ definido por $f^{\prime}(x)=\langle\operatorname{grad} f(x) \mid x\rangle$, onde $\langle 1\rangle$, denota o produto interno canônico de $\mathbb{R}^{\mathrm{n}}$. Obte-mos no final, uma classificação dos pares ( $\left.f, f^{\prime}\right)$ relativa-mente aos pares de pontos críticos associados. Usando o con ceito de k-decidibilidade apresentado em [3] determinamos uma classe de germes $f:\left(\mathbb{R}^{\mathrm{n}} ; 0\right) \stackrel{\mathrm{C}^{1}}{\longrightarrow}(\mathbb{R} ; 0)$ para a qual $f$ e $f^{\prime}$ têm o mesmo tipo de comportamento.

No capitulo III generalizamos os resultados obtidos:no capitulo II, classificando os pares $\left(f, f_{A}^{\prime}\right)$ relativamente - 
aos pares de pontos críticos associados, para $A \in M_{n}$ ( $\mathbb{R}$ ) simétrica, definida positiva, e $f_{A}^{\prime}:\left(\mathbb{R}^{n} ; 0\right) \longrightarrow(\mathbb{R} ; 0)$ defin $\underline{i}$ da por $f_{A}^{\prime}(x)=\langle\operatorname{gradf}(x) \mid A x\rangle$.

No ültimo capítulo fazemos uma ap1jcação à Estabilidade de Liapunov. Apresentamos uma demonstrção de um caso par ticular do Teorema de Liapunov para Instabilidade de sistemas mecânicos conservativos com dois graus de 1 iberdade, usando funções auxiliares do tipo Chetaev, que surgem naturalmente, motivadas pelos resultados dos capítulos II e III. Apresentamos também um exemplo com três graus de liberdade para mostrar a impossibilidade de generalizar tal demonstra ção para mais de dois graus de 1 iberdade.

Usaremos, no texto, o símbolo 8 no início e no final de cada demonstração apresentada. 


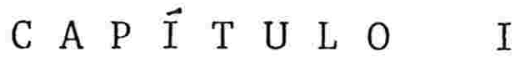

Uma demonstração elementar do Teorema Algébrico de Sard 


\section{I-1 INTRODUÇÃO}

Neste capítulo pretendemos apresentar alguns resultados já conhecidos sobre variedades algébricas, com o objetivo de aplicá-los ao estudo da geometria das seguintes variedades algébricas particulares:

$$
V(f)=\left\{x \in \mathbb{R}^{n} \mid \operatorname{grad} f(x) \text { é } \operatorname{radia} 1\right\}
$$

e

$$
V^{\perp}(f)=\left\{x \in \mathbb{R}^{n}|<\operatorname{grad} f(x)| x>=0\right\},
$$

onde $f$ é um polinômio em $n$ variáveis.

Usaremos frquentemente as seguintes notações:

$$
\begin{array}{ll}
\mathrm{S}_{\varepsilon}=\left\{\mathrm{x} \in \mathbb{R}^{\mathrm{n}}|| \mathrm{x} \mid=\varepsilon\right\} \quad, & \varepsilon>0 \\
\mathrm{~B}_{\varepsilon}=\left\{\mathrm{x} \in \mathbb{R}^{\mathrm{n}}|| \mathrm{x} \mid<\varepsilon\right\} \quad, & \varepsilon>0 \\
\mathrm{~B}_{\varepsilon}^{\prime}=\mathrm{B}_{\varepsilon}-\{0\} & \quad, \quad \varepsilon>0
\end{array}
$$

$f:\left(\mathbb{R}^{n} ; 0\right) \longrightarrow(\mathbb{R} ; 0)$ denotarâ um polinômio com $f(0)=0$.

\section{I-2 AS VARIEDADES ALGEBBRICAS $V(f)$ E $V^{\perp}(f)$}

$$
\begin{aligned}
\text { Sejam }: f:\left(\mathbb{R}^{\mathrm{n}} ; 0\right) & \longrightarrow(\mathbb{R} ; 0) \text { e } g:\left(\mathbb{R}^{\mathrm{n}} ; 0\right) \\
\mathrm{x} & \longrightarrow(\mathbb{R} ; 0)
\end{aligned}
$$

Def.I-2-1: Chamaremos $V(f)$ ao conjunto dos pontos de $\mathbb{R}^{\mathrm{n}}$ em que gradf è radial, is to é

$$
\begin{aligned}
V(f) & =\left\{x \in \mathbb{R}^{n} \mid \text { posto }\left[\begin{array}{l}
\operatorname{grad}(x) \\
\operatorname{gradg}(x)
\end{array}\right]<2\right\} \\
& =\left\{x \in \mathbb{R}^{n}|<\operatorname{grad} f(x)| x>^{2}=|\operatorname{gradf}(x)| \cdot|x|^{2}\right\}
\end{aligned}
$$


Def.I-2-2: Chamaremos $V^{\perp}(f)$ ao conjunto dos pontos de $\mathbb{R}^{n}$ em que gradf tem componente radial nula, isto é

$$
V^{\perp}(f)=\left\{x \in \mathbb{R}^{n} \mid\langle\operatorname{grad} f(x) \mid x\rangle=0\right\}
$$

Verifica-se imediatamente que $V(f) \cap V^{\perp}(f)$ ë exatamente o conjunto dos pontos críticos de f.

Def.I-2-3: Um subconjunto $S \subset \mathbb{R}^{\mathrm{n}}$ diz-se uma variedade algébri ca $\left(\right.$ de $\left.\mathbb{R}^{\mathrm{n}}\right)$ se existe uma família $\left\{\mathrm{P}_{\alpha}\right\}_{\alpha \in \Lambda}$, com $\mathrm{P}_{\alpha} \in \mathbb{R}\left[\mathrm{x}_{1}, \ldots, \mathrm{x}_{\mathrm{n}}\right]$, tal que

$S=\left\{x \in \mathbb{R}^{n} \mid P_{\alpha}(x)=0, \forall \alpha \in \Lambda\right\}$

Observemos que $V(f)$ e $V^{\perp}(f)$ são variedades algébricas de $\mathbb{R}^{\mathrm{n}}$ (contendo a origem) e assim, $V(f) \cap S_{\varepsilon}$ e $V^{\perp}(f) \cap S_{\varepsilon}$ são também variedades algébricas $(\varepsilon>0)$.

Alêm disso, como $V(f) \cap S_{\varepsilon}(\varepsilon>0)$ é exatamente o conjunto dos pontos crígicos de $\left.f\right|_{S_{\varepsilon}}$, segue que a origem não é ponto isolado de $V(f)$.

Ao contrário, $V^{\perp}(f)-\{0\}$ nem sempre é aderente à origem. Pode mesmo ocorrer que $\mathrm{V}^{\perp}(f)=\{0\}$, como mostra o seguinte

Exemp1o I-2-1:

$$
f(x)=\sum_{i=1}^{n} x_{i}^{2} \quad V^{\perp}(f)=\{0\}
$$


Apesar disto, quando $V^{\perp}(f)-\{0\} \vec{e}$ não vazio e adere à origem, temos para $V(f)$ e para $V^{\perp}(f)$ a mesma descrição geométrica local, proveniente do fato de ambas serem varieda-des algébricas de $\mathbb{R}^{n}$. Is to ficará mais claro no decorrer deste capítulo.

\section{I-3 DECOMPOSIÇÃO DE VARIEDADES ALGEBRICAS}

Def.I-3-1: Um ane1 A diz-se noetheriano se toda cadeia cres cente de ideais de A é estacionária (isto é, é finalmente constante).

Teor.I-3-1: (Teorema da base de Hilbert)

Se um ane1 A é noetheriano, então $A[x]$ è noethe riano.

(para uma demonstração deste resultado, ver [2])

Corolärio I-3-1a: Se A è um ane1 noetheriano, então $A\left[x_{1}, \ldots, x_{n}\right]$ é noetheriano.

Corolārio I-3-1b: Toda cadeia estritamente decrescente

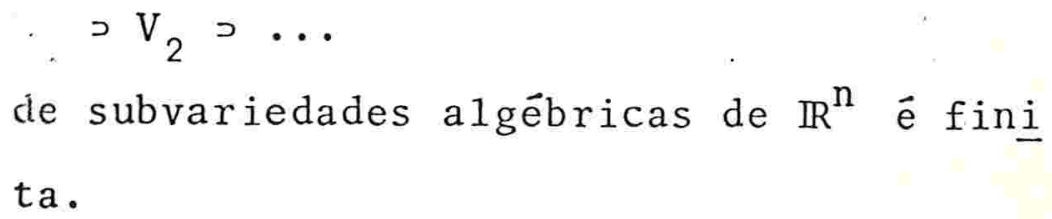$$
\supset \mathrm{V}_{2} \supset \ldots
$$$$
\text { de subvariedades algébricas de } \mathbb{R}^{\mathrm{n}} \text { é fin } \underline{i}
$$
ta.

(pois $\mathbb{R}$ é noetheriano) 
Seja $\left\{f_{\alpha}\right\}_{\alpha \in \Lambda}$ uma família em $\mathbb{R}\left[x_{1}, \ldots, x_{n}\right]$ e $V=\left\{x \in \mathbb{R}^{n} \mid f_{\alpha}(x)=0 \quad, \quad \forall \alpha \in \Lambda\right\}$

Prop.I-3-1: Existem $f_{1}, \ldots, f_{m} \in \mathbb{R}\left[x_{1}, \ldots, x_{n}\right]$ tais que

$$
V=\left\{x \in \mathbb{R}^{n} \mid f_{j}(x)=0 \quad, \quad 1 \leq j \leq m\right\}
$$

8 Consideremos o ideal de $\mathbb{R}\left[x_{1}, \ldots, x_{n}\right]$ definido por

$$
I(V)=\left\{f \in \mathbb{R}\left[x_{1}, \ldots, x_{n}\right] \mid f(x)=0, \quad \forall x \in V\right\}
$$

Segue do corolário I-3-1a que I(V) è finitamente gera do como $\mathbb{R}\left[x_{1}, \ldots, x_{n}\right]$-módulo.

Seja $f_{1}, \ldots, f_{m}$ um conjunto de geradores de $I(V)$. Então

$$
V=\left\{x \in \mathbb{R}^{n} \mid f_{i}(x)=0 \quad, \quad 1 \leq i \leq m\right\}
$$

De fato: i) $V \subset\left\{x \in \mathbb{R}^{n} \mid f_{i}(x)=0,1 \leq i \leq m\right\}$

ii) Para cada $\alpha \in \Lambda$, como $f_{\alpha} \in I(V)$, existem

$$
\begin{aligned}
& g_{1}, \ldots, g_{m} \in \mathbb{R}\left[x_{1}, \ldots, x_{n}\right] \text { tais que } \\
& f_{\alpha}=g_{1} f_{1}+\ldots+g_{m} f_{m}
\end{aligned}
$$

Logo $f_{\alpha}$ se anula onde $f_{1}, \ldots, f_{m}$ se anulam simulta neamente, donde

$$
V \supset\left\{x \in \mathbb{R}^{n} \mid f_{i}(x)=0,1 \leq i \leq m\right\}
$$

Sejam $V \subset \mathbb{R}^{\mathrm{n}}$ uma variedade algुébrica, $\left\{f_{1}, \ldots, f_{m}\right\}$ e $\left\{g_{1}, \ldots, g_{k}\right\}$ duas famílias de geradores de $I(V)$. Exis-tem $a_{i j} \in \mathbb{R}\left[x_{1}, \ldots, x_{n}\right], \quad 1 \leq i \leq k, 1 \leq j \leq m$, tais que

$$
g_{i}=\sum_{j=1}^{m} a_{i j} f_{j} \quad, \quad 1 \leq i \leq k
$$


Como $f_{j}(x)=0 \quad \forall x \in V, \quad 1 \leq j \leq m$, segue a identidade matricia1

$\left[\frac{\partial g_{i}(x)}{\partial x_{j}}\right]=\left[a_{i j}(x)\right]\left[\frac{\partial f_{i}(x)}{\partial x_{j}}\right], \quad \forall x \in V$

Logo ,

posto $\left[\frac{\partial g_{i}(x)}{\partial x_{j}}\right] \leq$ posto $\left[\frac{\partial f_{i}}{\partial x_{j}}(x)\right], \quad \forall \quad x \in V$

Isto mostra que, na verdade,

posto $\left[\frac{\partial g_{i}(x)}{x_{j}}\right]=$ posto $\left[\frac{\partial f_{i}}{\partial x_{j}}\right], \quad \forall \quad x \in V$

Este fa to nos permite dar as seguintes definições:

$\underline{\text { Def.I-3-2 }}: \rho=\max _{x \in V}\left\{\right.$ posto $\left.\left[\frac{\partial f_{i}}{\partial x_{j}}(x)\right]\right\}$ é dito posto de $I(V)$.

Def.I-3-3: $x \in V$ é dito um ponto não sigular (ou simples) de $V$ se posto $\left[\frac{\partial f_{i}}{\partial x_{j}}\right]=\rho$.

Caso conträrio, $\mathrm{x}$ é dito um ponto sigular de $\mathrm{V}$.

Notação: $M_{1}=M_{1}(V)$ é o conjunto dos pontos não sigulares de V.

Teorema I-3-2: Seja $V \subset \mathbb{R}^{\mathrm{n}}$ uma variedade algëbrica e $\rho=$ posto $(I(V))$.

Seja $M$ o conjunto dos pontos não sigulares de V. 
Então $V_{1}=V-M_{1}$ é também uma variedade a1gêbrica.

8 Sejam $f_{1}, \ldots, f_{m}$ geradores de $I(V)$

Observemos que

$$
\begin{aligned}
& V_{1}=\left\{x \in V \mid \text { posto }\left[\frac{\partial f_{i}}{\partial x_{j}}(x)\right]<\rho\right\} \\
& =\left\{x \in \mathbb{R}^{n} \mid f_{1}(x)=\ldots=f_{m}(x)=0 \text { e posto }\left[\frac{\partial f_{i}}{\partial x_{j}}(x)\right]<\rho\right\} \\
& =\left\{\begin{array}{r}
x \in \mathbb{R}^{n} \mid f_{1}(x)=\ldots=f_{m}(x)=0 \text { e } \frac{\partial\left(f_{i_{1}}, \ldots, f_{i_{\rho}}\right)(x)}{\partial\left(x_{\mu_{1}}, \ldots, x_{\mu_{\rho}}\right)} \\
1 \leq i_{j} \leq m, \quad 1 \leq \mu_{j} \leq n
\end{array}\right\} \text {. } \\
& \text { Como } \frac{\partial\left(f_{i_{1}}, \ldots, f_{i_{\rho}}\right)}{\left(x_{\mu}, \ldots, x_{\mu}\right)} \in \mathbb{R}\left[x_{1}, \ldots, x_{n}\right] \text { segue a tese } 8
\end{aligned}
$$

Nosso objetivo agora é demonstrar o seguinte

Teorema I-3-3: Se $V \subset \mathbb{R}^{\mathrm{n}} \overline{\mathrm{e}}$ uma variedade algébrica tal que posto $(I(V))=\rho$, então $M_{1}$ é uma subvariedade diferenciável analítica de $\mathbb{R}^{\mathrm{n}}$, de codimensão $\rho$.

Para demonstrarmos este resultado precisaremos de alguns lemas que desenvolveremos a seguir.

Fixemos $p \in M_{1}$ e $f_{1}, \ldots, f_{m}$ geradores de $I(V)$ tais que $f_{1}, \ldots, f_{\rho}$ tenham diferenciais linearmente indepen-dentes em $p$. 
Lema I-3-3a: Existe um sistema de coordenadas $\xi: U \rightarrow U_{0} \subset \mathbb{R}^{n}$ onde $U \subset \mathbb{R}^{\mathrm{n}}$ é uma vizinhança aberta de $\mathrm{p}$, tal que

$$
\xi_{i}=f_{i}-f_{i}(p) \quad \text { para } 1 \leq i \leq \rho
$$

$8 \quad$ Como $\mathrm{df}_{1}(\mathrm{p}), \ldots, \mathrm{df} \mathrm{f}_{\rho}(\mathrm{p})$ são 1 inearmente independen-tes, temos $\rho \leq n$,

Caso $\rho \mathrm{n}$, escolhemos vetores $\mathrm{w}_{\rho+1}, \ldots, \mathrm{w}_{\mathrm{n}} \in \mathbb{R}^{\mathrm{n}}$ tais que $\mathrm{df}_{1}(\mathrm{p}), \ldots, \mathrm{df_{ \rho }}(\mathrm{p}), \mathrm{w}_{\rho+1}, \ldots, \mathrm{w}_{\mathrm{n}}$ sejam 1 inearmente independentes e definimos
$(*)\left\{\begin{array}{l}\xi_{i}(x)=f_{i}(x)-f_{i}(p) \\ \xi_{i}(x)=\left\langle w_{i} \mid x-p\right\rangle\end{array}\right.$
$1 \leq i \leq \rho$
$\rho+1 \leq i \leq n$

Segue imediantamente do Teorema da Função Inversa que (*) define um sistema de coordenadas analítico numa vizi-nhança aberta $U$ de $p$ sobre uma vizinhança aberta $U_{0}$ de $0 \in \mathbb{R}^{\mathrm{n}}$.

Sejam $\xi: U \longrightarrow U_{0}$ como no lema anterior

e $\quad \Lambda=\left\{\lambda=\left(\lambda_{1}, \ldots, \lambda_{\rho+1}\right) \in \mathbb{N}^{\rho+1} \mid 1 \leq \lambda_{1}<\ldots<\lambda_{\rho+1} \leq n\right\}$

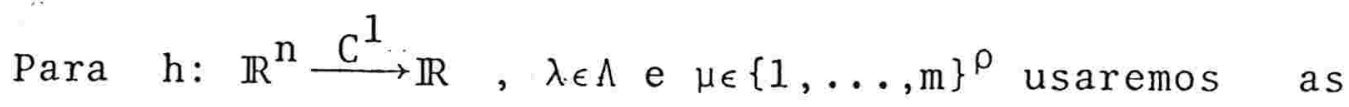
notações

$$
\begin{gathered}
\frac{\partial\left(F_{\mu}, h\right)}{\partial x_{\lambda}}=\frac{\partial\left(f_{\mu_{1}}, \ldots, f_{\mu}, h\right)}{\partial\left(x_{\lambda_{1}}, \ldots, x_{\lambda_{\rho}}, x_{\lambda_{\rho+1}}\right)}=\frac{\partial\left(F_{\mu}, h\right)}{\partial\left(x_{\lambda_{1}}, \ldots, x_{\lambda_{\rho}}, x_{\lambda_{\rho}+1}\right)} \\
\text { e } \quad \frac{\partial(F, h)}{\partial x_{\lambda}}=\frac{\partial\left(F_{\mu}, h\right)}{\partial x_{\lambda}} \text { quando } \mu=(1,2, \ldots, \rho)
\end{gathered}
$$


Para cada $\lambda \in \Lambda$ definimos o operador diferenciação

$$
\begin{aligned}
& \Phi_{\lambda}: \quad C^{\omega}(U) \longrightarrow C^{\omega}(U) \\
& \Phi_{\lambda} h=\frac{\partial(F, h)}{\partial x_{\lambda}}
\end{aligned}
$$

\section{Lema I-3-3b:}

$$
\Phi_{\lambda}(\mathrm{I}(\mathrm{V})) \subset \mathrm{I}(\mathrm{V}) \quad, \quad \forall \lambda \in \Lambda
$$

8 o resultado decorre imediatamente do fato de que se $h \in I(V), d f_{1}, \ldots d f_{\rho}$, dh são linearmente dependentes em ca da ponto de $V$, pois posto $(I(V))=\rho$.

Observemos que, por aplicações iteradas do lema anterior, podemos concluir que se $h \in I(V)$ então

$$
\begin{aligned}
{ }_{\lambda^{S}}{ }^{\Phi} \lambda^{S-1} \cdots{ }_{\lambda^{1}} h \in I(V) & \forall s \in \mathbb{N}^{*} \\
& \forall\left(\lambda^{1}, \ldots, \lambda^{s}\right) \in \Lambda^{s}
\end{aligned}
$$

Fixemos $g \in \mathrm{I}(V)$

Seja $\mathrm{Hg}(U)$ o ideal de funções analíticas em U gerado pela familia

$$
\left\{\begin{array}{rll}
\left\{\Phi_{i s}\right. & \cdots & \Phi_{\lambda^{1}} g
\end{array}\right\}_{s \in \mathbb{N}^{*}}
$$

Lema I-3-3c: $\Phi_{\lambda}(H g(U)) \subset H g(U), \forall \lambda \in \Lambda$.

$8 \quad \operatorname{Seja} h \in H g(U)$. Então

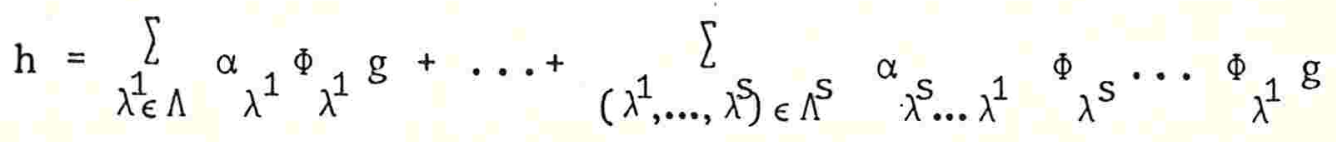


Onde $\quad{ }_{\lambda}{ }_{\lambda} \ldots \lambda^{2} \lambda^{1} \in C^{\omega}(U) \quad, \quad 1 \leq j \leq s$

Como $\Phi_{\lambda}$ é um operador derivação segue a tese.

Lema I-3-3d: Se $h \in H g(U)$, então

$$
\frac{\partial \mathrm{h}}{\partial \xi_{\mathrm{k}}} \in \mathrm{Hg}(\mathrm{U}) \quad, \quad \forall \mathrm{k} \in\{\rho+1, \ldots, \mathrm{n}\}
$$

8 Lembremos que $\xi: U \longrightarrow U_{0}$ satisfaz

$$
\xi_{i}=f_{i}-f_{i}(p) \quad, \quad 1 \leq i \leq \rho
$$

Disto segue que, para $k \in\{\rho+1, \ldots, n\}$

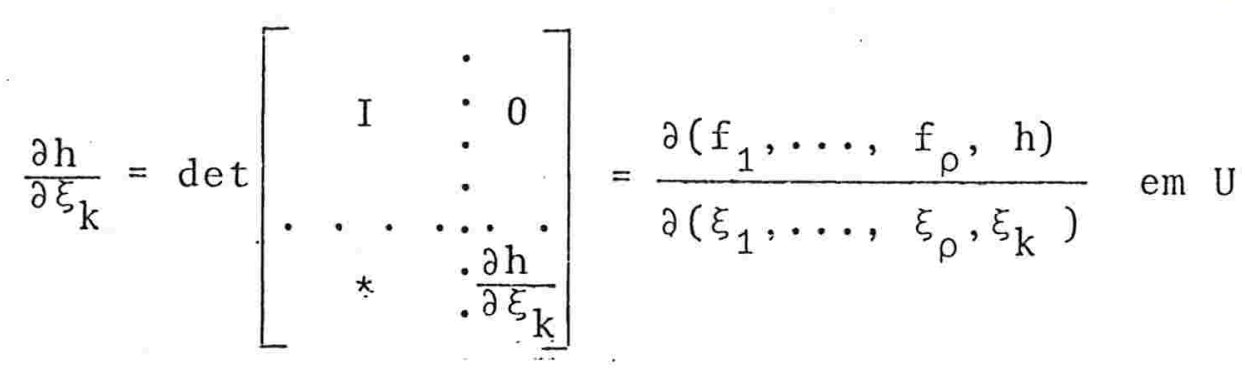

Aplicando a identidade de Lagrange obtemos

$$
\begin{aligned}
\frac{\partial\left(f_{1}, \ldots, f_{\rho}, h\right)}{\partial\left(\xi_{1}, \ldots, \xi_{\rho}, \xi_{k}\right)} & =\sum_{\lambda \in \Lambda} \frac{\partial\left(f_{1}, \ldots, f_{\rho}, h\right)}{\partial x_{\lambda}} \frac{\partial x_{\lambda}}{\partial\left(\xi_{1}, \ldots, \xi_{\rho}, \xi_{k}\right)} \\
& =\sum_{\lambda \in \Lambda}\left(\Phi_{\lambda} h\right) \frac{\partial x_{\lambda}}{\partial\left(\xi_{1}, \ldots, \xi_{\rho}, \xi_{k}\right)}
\end{aligned}
$$

Como $\frac{\partial x_{\lambda}}{\partial\left(\xi_{1}, \ldots, \xi_{\rho}, \xi_{k}\right)}$ è analítico em $U$ (pois $\xi \hat{e}$ um sistema de coordenadas analítico) temos $\frac{\partial h}{\partial \xi_{k}} \in H g(U) \cdot \quad 8$ 
Segue do lema anterior que as derivadas parciais de $g$ de qualquer ordem com respeito às coordenadas $\left(\xi_{p+1}, \ldots, \xi_{n}\right)$ estão em $\mathrm{Hg}(U)$.

Prova do Teorema I-3-3:

$8 \quad$ Seja $M^{*}=\left\{q \in U \mid f_{1}(q)=\ldots=f_{\rho}(q)=0\right\}$

$M^{*}$ é uma subvariedade diferenciāvel analítica de $\mathbb{R}^{\mathrm{n}}$ de codimensão $\rho$, com sistema de coordenadas $\left(\xi_{\rho+1}, \ldots, \xi_{n}\right)$, e $\mathrm{p} \in \mathrm{M}^{*}$.

Diminuindo U se necessärio, podemos supor que $M^{*}$ é cọ nexa.

Seja $\tau=M_{1} \cap U$.

Observemos que

$\tau=M_{1} U=V \cap U=\left\{q \in U \mid f_{1}(q)=\ldots=f_{\rho}(q)=\ldots f_{m}(q)=0\right\} \subset M^{*}$

Vamos provar que $\tau \supset M^{*}$, e, consequentemente, que $\tau=M_{1} \cap U=M^{*}$, donde concluiremos a tese, pois $p \in M$ foi to mado arbitrariamente.

Para mostrar que $\tau \supset M^{*} \vec{e}$ suficiente provar que $\left.g\right|_{M^{*}}=0$, pois g foi tomada arbitrariamente em I(V). Por outro lado, como g é analítica em $U$ e $M^{*}$ é uma subvariedade diferenciâa vel analitica, conexa, contida em U, com sistema de coorde nadas $\left(\xi_{p+1}, \ldots, \xi_{n}\right)$, se mostrarmos que as derivadas parciais de $g$ de qualquer ordem com respeito a $\xi_{\rho+1}, \ldots, \xi_{n}$ se anulam em $\mathrm{p}^{\mathrm{M}} \mathrm{M}^{*}$ teremos $\left.\mathrm{g}\right|_{\mathrm{M}^{*}}=0$, pois $\mathrm{g}(\mathrm{p})=0$. 
Pelo lema I-3-3d, é suficiente provar que se $h \in H g(U)$ então $h(p)=0$.

Tomemos $\mathrm{h} \in \mathrm{Hg}(\mathrm{U})$

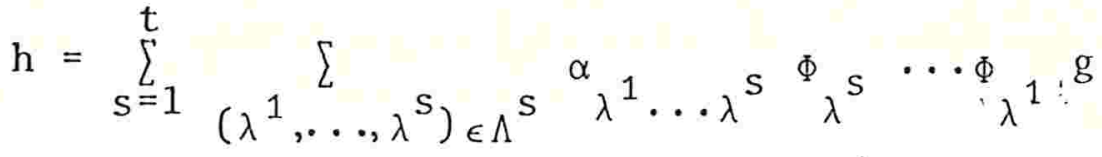

$$
\begin{aligned}
& \text { onde } \alpha_{\lambda^{1} \ldots \lambda^{\mathrm{s}}} \mathrm{C}^{\omega}(\mathrm{U}) \quad, \quad 1 \leq \mathrm{s} \leq \mathrm{t} \\
& \text { Como } \Phi_{\lambda} \mathrm{s} \cdots \Phi_{\lambda^{1}} \mathrm{~g} \in \mathrm{I}(\mathrm{V}) \quad \text { (pelo 1.ema I-3-3b) } \\
& \text { e } \quad p \in M_{1} \quad \text { temos } h(p)=0
\end{aligned}
$$

Teor.I-3-4: Se $V \subset \mathbb{R}^{\mathrm{n}}$ é uma variedade algëbrica, então

$$
\begin{aligned}
& V=M_{1} \cup M_{2} \cup \quad \cdots u \cdot M_{k} \\
& \text { onde a união é disjunta e } M_{i}(1 \leq i \leq k) \text { é uma } \\
& \text { subvariedade diferenciävel analítica de } \mathbb{R}^{n} \text {. }
\end{aligned}
$$

$\stackrel{\nabla}{\Delta}$

Seja $\rho=$ posto $(\mathrm{I}(\mathrm{V}))$

Se $\rho=0$ então $I(V)=0$ e $\quad V=\mathbb{R}^{n}$

Suponhamos então que $\rho>0$

Chamando $V_{0}=V$, definimos indutivamente, para $i \in \mathbb{N}^{*}$ :

$$
\begin{aligned}
& M_{i}=M_{1}\left(V_{i-1}\right)=\left\{x \in V_{i-1} \mid x \text { é não singular }\right\} \\
& V_{i}=V_{i-1}-M_{i}
\end{aligned}
$$

(observemos que a definição acima tem sentido, pois segue do teorema I-3-2 que $V_{i} \subset \mathbb{R}^{n}$ è uma variedade algêbrica, o que nos permite definir $M_{i+1}$ ) 
Obtemos assim uma cadeia decrescente de variedades a1 gëbricas

$$
\mathrm{v}_{0} \supset \mathrm{V}_{1} \supset \ldots>\mathrm{V}_{\mathrm{m}} \supset \ldots
$$

que deve ser estacionäria, pelo corolärio I-3-1b.

Seja $k \in \mathbb{N}^{*}$ satisfazendo

(i) $\quad \mathrm{v}_{\mathrm{k}}=\mathrm{v}_{\mathrm{k}+1}$

(ii) $\mathrm{v}_{\mathrm{k}-1} \neq \varnothing$

Observemos que $\mathrm{V}_{\mathrm{k}}=\varnothing$, caso conträrio teríamos

$M_{k+1}=M_{1}\left(V_{k}\right) \neq \varnothing$ e consequentemente

$\mathrm{v}_{\mathrm{k}+1}=\mathrm{V}_{\mathrm{k}}-\mathrm{M}_{\mathrm{k}+1} \nsucceq \mathrm{V}_{\mathrm{k}}$, contra a escolha de $\mathrm{k}$.

Logo,

$v_{j}=\varnothing \quad \forall j \geq k \quad$ e $\quad M_{k}=V_{k-1}$

Mas então:

$$
\begin{aligned}
V=V_{0} & =M_{1} \cup\left(V_{0}-M_{1}\right)=M_{1} \cup V_{1} \\
& =M_{1} \cup \quad M_{2} \cup \quad V_{2}=\ldots \\
& =M_{1} \cup M_{2} \cup \ldots \cup M_{k},
\end{aligned}
$$

sendo tal união disjunta.

Como $M_{i}=M_{1}\left(V_{i-1}\right), 1 \leq i \leq k$, pe1o teorema I-3-3 segue a tese. 
Passemos agora a desenvolver alguns resultados para demonstrar o

Teor.I-3-5: Se $V \subset \mathbb{R}^{\mathrm{n}}$ ë uma variedade algébrica, então $V$ po de ser decomposta numa união finita de subva-riedades diferenciảveis analíticas conexas, disjuntas, de $\mathbb{R}^{\mathrm{n}}$.

A idéia é mostrar que $M_{1}=M_{1}(V)$ tem um nümero finito de componentes conexas, e então usar o teorema I-3-4.

Lema I-3-5a: Sejam $g \in \mathbb{R}\left[\underline{-1}_{1}, \ldots, x_{n}\right] \quad, f_{1}, \ldots, f_{m}$ geradores de I(V) e $\rho=$ posto $(I(V))$.

0 conjunto $\Gamma$ dos pontos críticos de $\left.g\right|_{M_{1}}$ fica definido por

$$
\Gamma=M_{1} \cap\left\{x \in \mathbb{R}^{n} \mid \frac{\partial\left(F_{\mu}, g\right)}{\partial x_{\lambda}}(x)=0, \forall \mu \in\{1, \ldots, m\}^{\rho}, \forall \lambda \in \Lambda\right\}
$$

$8 \quad M_{1}$ è uma subvariedade diferenciävel de $\mathbb{R}^{\mathrm{n}}$ de codimen são $\rho, e \cdot d f_{1}(x), \ldots, d f_{m}(x) \operatorname{geram}\left(T_{x} M_{1}\right)^{\perp}, \quad \forall \quad x \in M_{1}$.

Como $x \in M$ é ponto crítico de $\left.g\right|_{M_{1}} \Longleftrightarrow d g(x) \in\left(T_{x_{1} M_{1}}\right)^{\perp}$

$$
\Longleftrightarrow \rho=\operatorname{posto}\left[\begin{array}{l}
\mathrm{d}_{1}(x) \\
\mathrm{d} f_{m}(x)
\end{array}\right]=\operatorname{posto}\left[\begin{array}{c}
\mathrm{d} f_{1}(x) \\
\dot{\dot{x}} \\
\mathrm{~d} \dot{f}_{m}(x) \\
\mathrm{dg}(x)
\end{array}\right]
$$


Lema I-3-5b: Se $f_{1}, \ldots, f_{m}$ são geradores de $I(V)$ e em $x^{0} \in V$ a matriz $\left[\frac{\partial f_{i}}{\partial x_{j}}\right]$ tem posto $n$, então $V-\left\{x^{\circ}\right\}$ é ainda uma variedade algébrica. Em particu-lar, $x^{0}$ é um ponto isolado de $\mathrm{V}$.

8 Sem perda de generalidade, podemos supor que $x^{\circ}=0$ e que $\mathrm{df}_{1}(0), \ldots, \mathrm{df} \mathrm{n}_{\mathrm{n}}(0)$ são linearmente independentes.

Como $f_{j}(0)=0, \quad 1 \leq j \leq n$, existem $g_{j i} \in \mathbb{R}\left[x_{1}, \ldots, x_{n}\right]$, $1 \leq i, j \leq n$, tais que

$$
f_{j}(x)=g_{j 1}(x) x_{1}+g_{j 2}(x) x_{2}+\ldots+g_{j n}(x) x_{n}, \forall x \in \mathbb{R}^{n}
$$

Seja $W \subset V$ a variedade algëbrica definida por

$\left\{\begin{array}{l}f_{1}(x)=f_{2}(x)=\ldots=f_{m}(x)=0 \\ \operatorname{det}\left[g_{j k}(x)\right]=0\end{array}\right.$

$x^{0}=0 \notin W$, pois a matriz

$$
\left[\frac{\partial f_{j}}{\partial x_{k}}(0)\right]_{1 \leq j, k \leq n}=\left[g_{j k}(0)\right] \text { è não singular }
$$

Por outro lado, se $x \in V$ é não nulo, temos

$$
\left[\begin{array}{c}
0 \\
\dot{0} \\
\dot{0}
\end{array}\right]=\left[\begin{array}{c}
f_{1}(x) \\
\dot{f_{n}}(x)
\end{array}\right]=\left[\begin{array}{c}
g_{11}(x) \\
\vdots \\
g_{n 1}(x)
\end{array}\right] x_{1}+\ldots+\left[\begin{array}{c}
g_{1 n}(x) \\
\vdots \\
g_{n n}(x)
\end{array}\right] x_{n}
$$

e portanto, as colunas da matriz $\left[g_{j k}(x)\right]$ são linearmente dependentes, donde $x \in W$. Logo, $\mathbb{W}=V-\left\{x^{\circ}\right\}$ 
Corolärio: Se V tem dimensão zero, então V é finito.

8 Podemos supor que $V \neq \varnothing$

Sejam $f_{1}, \ldots, f_{m}$ geradores de $I(V)$ e $\rho=$ posto $(\mathrm{I}(\mathrm{V}))$.

E claro que $\rho=n$, caso contrārio, pelo teorema I-3-3 $\mathrm{V}$ conteria uma subvariedade diferenciāvel de dimensão $n-\rho \geq 1$, contra o fato de $V$ ter dimensão zero.

Seja $\quad x^{0} \epsilon M_{1}=M_{1}(V)$

Então a matriz $\left[\frac{\partial f_{i}}{\partial x_{j}}\left(x^{0}\right)\right]$ tem posto $\rho=n$, donde segue do lema anterior, que $\mathrm{W}_{1}=\mathrm{V}-\left\{\mathrm{x}^{0}\right\}$ e uma variedade algébrica.

Notemos que, como $W_{1} c V, W_{1}$ tem tambẻm dimensão zero.

Chamando $W_{0}=V$, podemos repetir o raciocínio anterior para construir uma cadeia estritamente decrescente de va-riedades algébricas, a saber:

Se $W_{i} \neq \emptyset$, como $W_{i}$ tem dimensão zero, existe $x^{i} \in W_{i}$ tal que

$w_{i+1}=w_{i}-\left\{x^{i}\right\}$ e novamente uma variedade algébrica de dimensão zero.

Pelo corolārio I-3-1b, $\exists k \in \mathbb{N}$ tal que $W_{k+1}=\varnothing$ Logo, $V=\left\{x^{0}, x^{1}, \ldots, x^{k}\right\}$ 
Lema I-3-5c: Se $M_{1}=M_{1}(V)$ coincide com V, então $V$ tèn no máximo um nümero finito de componentes cọ nexas.

8 Um lema de Andreotti e Frankel garante que para quase toda escolha de $a \in \mathbb{R}^{\mathrm{n}}$, a função

$$
\begin{aligned}
r_{a}: V=M_{1} & \longrightarrow \mathbb{R} \\
x & \longrightarrow r_{a}(x)=|x-a|^{2}
\end{aligned}
$$

tem somente pontos críticos não degenerados.

Fixemos um ta $1 \quad a \in \mathbb{R}^{\mathrm{n}}$.

Seja

$\tau=\left\{x \in V \cdot \mid x\right.$ è um ponto crítico de $\left.\mathrm{r}_{\mathrm{a}}\right\}$

Tomemos $f_{1}, \ldots, f_{m}$ geradores de $I(V)$ e

seja $\rho=\operatorname{posto}(I(V))$.

Pelo lema I-3-5a,

$\tau=M_{1} \cap\left\{x \in \mathbb{R}^{n} \mid \frac{\partial\left(F_{\mu}, r_{a}\right)}{\partial x_{\lambda}}(x)=0 \quad, \quad \forall \mu \in\{1, \ldots, m\}^{\rho}, \forall \lambda \in \Lambda\right\}$

Como $M_{1}=V$ temos

$$
\begin{aligned}
\tau=\left\{x \in \mathbb{R}^{n} \mid\right. & f_{j}(x)=0, \quad 1 \leq j \leq m, \\
& \text { e } \left.\frac{\partial\left(F_{\mu}, r_{a}\right)}{\partial x_{\lambda}}(x)=0, \forall \mu \in\{1, \ldots, m\}^{\rho}, \forall \lambda \in \Lambda\right\}
\end{aligned}
$$

Assim, t é uma variedade algébrica.

Como pontos críticos não degenerados são sempre isola 
dos, segue que $\tau$ tem. dimensão zero, e portanto, pelo coro1ario do lema I-3-5b, $\tau$ é finito.

Agora observemos que cada componente conexa $\mathrm{V}^{\mathrm{i}}$ de $\mathrm{V} e \overrightarrow{\mathrm{e}}$ um fechado (pois $M_{1}=V$ è uma subvariedade diferenciāvel de $\mathbb{R}^{n}$ fechada). Mais ainda, $V^{i} \vec{e}$ uma subvariedade diferenciä vel conexa, fechada, sem bordo. Logo, ra $\mathrm{f}_{\mathrm{i}}$ assume mínimo num ponto $x^{i} \epsilon V^{i}$ e este ponto é ponto crítico de $\left.r_{a}\right|_{V}$.

Portanto, o número de. componentes conexas de $\mathrm{V}$ é menor ou igual a \# $\tau$.

Corolärio: Sejam $V \subset \mathbb{R}^{n}$ e $W \subset \mathbb{R}^{n}$ variedades algëbricas tais que os pontos sigulares de $V$ pertencem a $W$. Então V-W tem um número finito de componentes conexas, cada uma das quais é uma subvariedade di ferenciāvel analítica de $\mathbb{R}^{\mathrm{n}}$.

8 Sejam $g_{1}, \ldots, g_{k}$ geradores de $I(W)$

Definimos

$s(x)=g_{1}^{2}(x)+\ldots+g_{k}^{2}(x)$

Observemos que $\mathrm{s}(\mathrm{x})=0 \Longleftrightarrow \mathrm{x} \in \mathrm{W}$.

Consideremos agora a função racional

$$
\begin{aligned}
g=\frac{1}{s}: & V-W \\
x & \longrightarrow \mathbb{R} \\
& \longrightarrow g(x)=\frac{1}{s(x)}
\end{aligned}
$$

g estā bem definida e è contínua.

Seja G o gräfico de g. Temos: 


$$
\begin{aligned}
G & =\left\{(x, y) \mathbb{R}^{n} x \mathbb{R} \mid x \in V-W \text { e } \quad y=\frac{1}{s(x)}\right\} \\
& =\left\{(x, y) \mathbb{R}^{n} x \mathbb{R} \mid x \in V \text { e } s(x) y=1\right\}
\end{aligned}
$$

Tomemos $f_{1}, \ldots, f_{m}$ geradores de $I(V)$, e seja $\rho=$ posto $(I(V))$

Definindo

$\mathrm{F}_{i}: \mathbb{R}^{\mathrm{n}+1}=\mathbb{R}^{\mathrm{n}} \times \mathbb{R} \longrightarrow \mathbb{R}$ por

$$
F_{i}(x, y)=f_{i}(x) \quad, \quad 1 \leq i \leq m
$$$$
F_{m+1}(x, y)=s(x) y-1
$$

temos

$$
F_{i} \in \mathbb{R}\left[x_{1}, \ldots, x_{n}, y\right] \quad, \quad 1 \leq i \leq m+1 \quad \text { e }
$$

G ề definido por

$$
F_{1}(x, y)=F_{2}(x, y)=\ldots=F_{m+1}(x, y)=0
$$

Logo, $G \subset \mathbb{R}^{n+1}$ è uma variedade algébrica em V-W.

Como $V-W \subset M_{1}(V)$, a matriz $\left\lceil\frac{\partial f_{i}}{\partial x_{j}}\right]$ tem posto constante

Por outro lado, $\forall(x, y) \in G$ temos

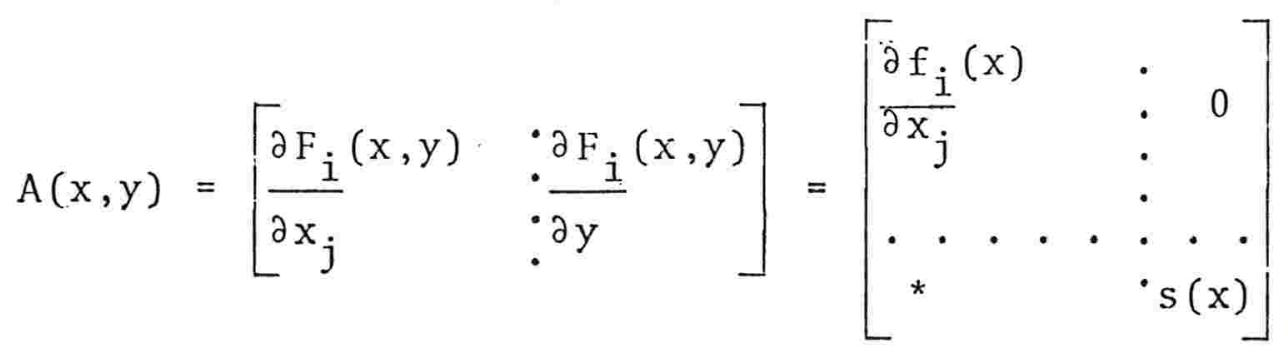

Logo, A tem posto constante $\rho+1$ em $G$ (pois se $(x, y) \in G$ então $x \in V-W$ e $s(x) \neq 0)$. 
Assim, $M_{1}(G)=G$ e segue do lema $I-3-5 c$ que $G$ tem um número finito de componentes conexas.

$$
\begin{aligned}
& \text { Como a 'projeçãd' } \\
& \text { ๆ }: \mathbb{R}^{n+1} x \mathbb{R} \longrightarrow \mathbb{R}^{n} \\
& (x, y) \longmapsto x
\end{aligned}
$$

é um difeomorfismo de G sobre $V-W$, segue a tese.

Prova do Teor. I-3-5:

8 Consideremos a decomposição

$$
V=M_{1} \cup M_{2} \cup \quad \ldots u v M_{k}
$$

de $V$ fornecida pelo teorema I-3-4.

Da definição de $M_{i}, 1 \leq i \leq k$, segue do corolário anterior que $M_{i}$ tem um número finito de componentes conexas.

Teor.I-3-6: Dadas duas variedades algëbricas $\mathbb{W} \subset \mathbb{R}^{\mathrm{n}} \quad$ e $V \subset \mathbb{R}^{n}$, a diferença $V-W$ tem um nünero finito de componentes conexas, sendo cada uma reunião finita dis junta de subvariedades diferenciäveis conexas de $\mathbb{R}^{\mathrm{n}}$.

8 Consideremos a decomposição de $V$

$$
V=M_{1} \cup M_{2} \cup \ldots \cup \quad M_{k}
$$

dada pelo teorema $\mathrm{I}-3-4$.

Temos

$V-W=\left(M_{1}-W\right) \cup \ldots u \quad\left(M_{k}-W\right) \quad$ e $\quad M_{i}-W=V_{i-1}-\left(V_{i} \cup W\right)$ 
Logo, $M_{i}-W$ é a diferença entre duas variedades a1gêbricas $V_{i-1}$ e $V_{i} \cup W$, sendo que $V_{i} u W$ contêm os pontos singulares de $V_{i-1}$ Segue do corolârio do lema $\mathrm{I}-3-5 \mathrm{c}$ que $\mathrm{M}_{\mathrm{i}}-\mathrm{W}$ tem um número finito de componentes conexas, sendo ca da uma subvariedade diferenciável analítica de $\mathbb{R}^{n}$.

I-4 ALGUMAS PROPRIEDADES DE V(f). E DE $\dot{V}^{\perp}(f)$

Teor.I-4-1 (Teorema Algébrico de Sard):

Se $V \subset \mathbb{R}^{n}$ é uma variedade algēbrica e $g \in \mathbb{R}\left[x_{1}, \ldots, x_{n}\right]$, então o conjunto dos valores críticos $\left.g\right|_{V}$ é finito.

8 Seja $\Gamma \subset V$ o conjunto dos pontos críticos de $g !_{V}$, e

(1) $V=M_{1} \cup M_{2} \cup \cdots \cup M_{k}$ decomposição de $V$ dada pelo teorema I-3-4.

(2) $\Gamma=\Gamma_{1} \cup \Gamma_{2} \cup \ldots \cup \Gamma_{k}$ onde $\Gamma_{i}=\Gamma \cap M_{j}, 1 \leq i \leq k$

Temos $c(\Gamma) \leq c\left(\Gamma_{1}\right)+c\left(\Gamma_{2}\right)+\ldots+c\left(\Gamma_{k}\right)$, onde $c(\Gamma) e ́ o$ número de componentes conexas de $\Gamma$ e $c\left(\Gamma_{i}\right)$ é o número de componentes conexas de $\Gamma_{i}, 1 \leq i \leq k$

Fixemos $i \in\{1, \ldots, k\}$.

Vamos mostrar que $c\left(\Gamma_{i}\right)$ é finito

Observemos que, decorre de (1) e (2) que $\Gamma_{i}$ é o con junto dos pontos críticos de $\left.g\right|_{M_{i}}$, e $M_{i}$ é o conjunto 
dos pontos não singulares de uma variedade algébrica $v_{i-1} \subset \mathbb{R}^{n}$ (ver prova do teorema $I-3-4$ ).

Sejam $f_{1}, \ldots, f_{m}$ geradores de $I\left(V_{i-1}\right)$ e $\rho=\operatorname{posto}\left(I\left(V_{i-1}\right)\right)$

Pelo lema I-3-5a

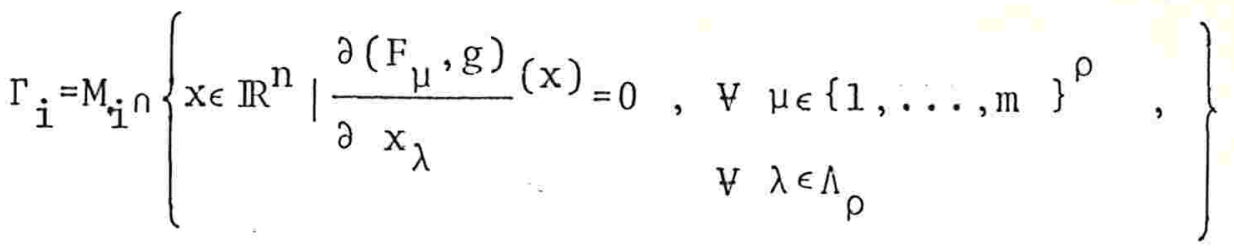

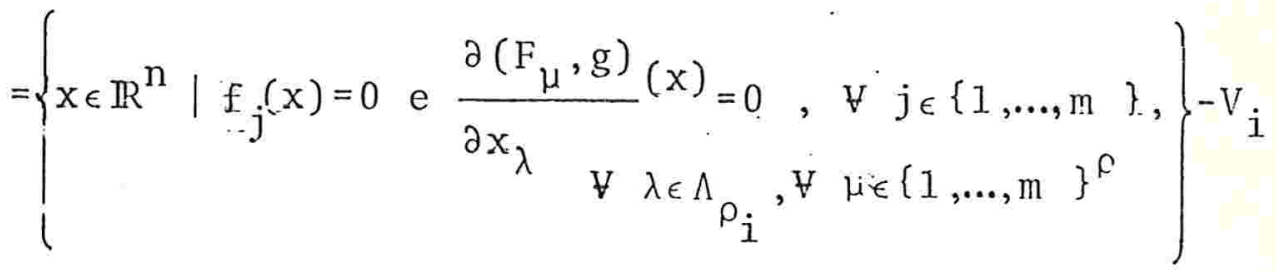

(onde $V_{i}=V_{i-1}-M_{i}$ )

Pelo teorema I-3-6, como $\Gamma_{i}$ é a diferença de duas variedades algébricas de $\mathbb{R}^{\mathrm{n}}$, segue que $c\left(\Gamma_{i}\right)$ é finito e que cada componente conexa de $\Gamma_{i} \hat{e}$ uma reunião finita dis junta de subvariedades diferenciâveis analíticas conexas de $\mathbb{R}^{\mathrm{n}}$.

Assim, $c(\Gamma)$ é finito e $\Gamma$ é a reunião finita disjunta de variedades diferenciáveis conexas, em cada uma das quais gé constante. 
Prop.I-4-1 : Se $f:\left(\mathbb{R}^{n} ; 0\right) \longrightarrow(\mathbb{R} ; 0)$ for um polinômio, en tão existe $\varepsilon_{0}>0$ tal que $V(f)$ é transversal a $S_{\varepsilon}, \forall \varepsilon \in\left(0, \varepsilon_{0}\right)$.

8 Seja $g:\left(\mathbb{R}^{n} ; 0\right) \longrightarrow(\mathbb{R} ; 0)$ o polinômio definido por $g(x)=|x|^{2}$.

Como $V(f)$ é uma variedade algébrica de $\mathbb{R}^{\mathrm{n}}$, segue do Teorenia Algëbrico de Sard que $\left.g\right|_{V(f)}$ tem um número finito de valores críticos. Seja $r$ o menor valor critico estritamente positivo de $\left.g\right|_{V(f)}$, e tomemos $\varepsilon_{0}=\sqrt{r}$

Então, se $x \in V(f) \cap S_{\varepsilon}$ (para $0<\varepsilon<\varepsilon_{0}$ ) temos que $x$ não é ponto crítico de $g !_{V(f)}$. Isto significa que a componente conexa da decomposição feita em I-3 para $V(f)$ que contém $x$ ê uma variedade diferenciāvel que não tangencia $S_{\varepsilon}$ em $x$, ou seja, que é transversal a $S_{\varepsilon}$ em $x$.

Com um raciocínio análogo concluimos:

Prop.I-4-2: Se $f:\left(\mathbb{R}^{\mathrm{n}} ; 0\right) \longrightarrow(\mathbb{R} ; 0)$ for um polinômio, então existe $\varepsilon_{0}>0$ tal que $V^{\perp}(f)$ è transver-sal a $S_{\varepsilon}, \quad \forall \varepsilon \in\left(0, \varepsilon_{0}\right)$.

Decorre da proposição I-4-1 que $V(f) \cap B_{\varepsilon_{0}^{\prime}}$ tem um número finito de componentes conexas, cada uma das quais é transversal às esferas $S_{\varepsilon}, \quad 0<\varepsilon<\varepsilon_{0}$.

Da proposição I-4-2 decorre resultado análogo para $V^{\perp}(f) \cap B_{\varepsilon_{0}^{\prime}}^{\prime}$. 
Prop.I-4-3: Se $V^{\perp}(f)-\{0\}$ não adere à origem, então a ori gem é ponto de extremo estrito de f.

Esta proposição voltará a ser mencionada no capítulo II de maneira mais geral, quando será demonstrada.

Agora vamos obter um resultado sobre separação

Prop.I-4-4: Seja $\mathrm{f}:\left(\mathbb{R}^{\mathrm{n}} ; 0\right) \longrightarrow(\mathbb{R} ; 0)$ um polinômio.

$$
\begin{aligned}
& \text { Se } x, y \in V(f) \text { satisfazem } \\
& \langle\operatorname{grad} f(x) \mid x\rangle\langle\operatorname{grad} f(y) \mid y\rangle<0, \\
& \text { então } V^{\perp}(f) \text { separa } x \text { de } y .
\end{aligned}
$$

8 Para toda curva $n:[0,1] \stackrel{C^{0}}{\longrightarrow} \mathbb{R}$ tal que

$$
\begin{aligned}
& n(0)=x \quad \text { e } n(1)=y \quad: \quad \text { a função } \\
& F_{n}:[0,1] \longrightarrow \mathbb{R}^{n} \\
& t \longmapsto
\end{aligned}
$$

è contínua e satisfaz

$$
\begin{aligned}
& F_{\eta}(0) \cdot F_{\eta}(1)<0 \\
& \text { Logo existe } \bar{t} \in(0,1) \text { tal que } F_{\eta}(\bar{t})=0 \\
& \text { Como } F_{\eta}(\bar{t})=\langle\operatorname{grad} f(\eta(\bar{t})) \mid \eta(\bar{t})\rangle \text { segue que } \\
& \eta(\bar{t}) \in V^{\perp}(f)
\end{aligned}
$$

Da arbitrariedade de $n$ segue a tese.

Corolârio: Seja $f:\left(\mathbb{R}^{\mathrm{n}} ; 0\right) \longrightarrow(\mathbb{R} ; 0)$ um polinômio. Se $\sigma_{1}$ e $\sigma_{2}$ são duas componentes de $V(f) \cap B \varepsilon_{0}^{\prime}$ 
(onde $\varepsilon_{0} \vec{e}$ dado pela prop.I-4-1) tais que $<\operatorname{gradf}(x) \mid x><0 \quad, \quad \forall x \in \sigma_{1}, \quad e$ $<\operatorname{gradf}(x) \mid x><0 \quad, \quad \forall x \in \sigma_{2}$, então $V^{\perp}(f)$ separa $\sigma_{1}$ de $\sigma_{2}$. 


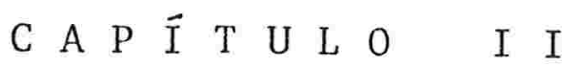

A derivada radial 


\section{I - 1 INTRODUÇÃO}

No presente capitulo abordaremos o problema de relacio nar o tipo de ponto crítico de uma função

$$
\mathrm{f}: \mathrm{U} \stackrel{\mathrm{C}^{1}}{\rightarrow} \mathbb{R}
$$

(onde $U \subset \mathbb{R}^{\mathrm{n}}$ é uma vizinhança aberta de $0 \in \mathbb{R}^{\mathrm{n}}$ e 0 é o ponto crítico de f) com o tipo de ponto crítico de sua derivada radial $f^{\prime}$, definida abaixo.

Sob este aspecto, obteremos, no final, uma classificação completa dos pares $\left(f, f^{\prime}\right)$.

$f:\left(\mathbb{R}^{\mathrm{n}} ; 0\right) \stackrel{\mathrm{C}^{\mathrm{k}}}{\longrightarrow}\left(\mathbb{R}^{\mathrm{m}} ; 0\right)$ denotarä germe de função $\mathrm{C}^{\mathrm{k}}$ na origem, e, como é usual, denotaremos também por $f$ um representante do germe.

Def.II-1-1 : $f:\left(\mathbb{R}^{\mathrm{n}} ; 0\right) \stackrel{\mathrm{C}^{\mathrm{O}}}{\longrightarrow}(\mathbb{R} ; 0)$.

Diremos que $f$ tem mảximo forte (mínimo forte) na origem se a origem for ponto de mäximo (mini mo) estrito de $f$.

Diremos que $f$ tem mäximo brando (mïnimo brando) na origem se a origem for ponto de máximo (míni mo) não estrito de $f$.

Diremos que $f$ tem sela na origem se a origem não. for ponto nem de mảximo nem de mínimo de $f$.

Def.II-1-2: $f:\left(\mathbb{R}^{\mathrm{n}} ; 0\right) \longrightarrow(\mathbb{R} ; 0)$

Diremos que o germe $f$ admite jato punctual de 
ordem $k$ na origem se existir um polinômio

$\mathrm{p}: \mathbb{R}^{\mathrm{n}} \longrightarrow \mathbb{R}$

de grau menor ou igual a $k$ tal que

$$
\lim _{x \rightarrow 0} \frac{f(x)-P(x)}{|x|^{k}}=0
$$

Observemos que $P$, quando existir, è único, e no caso de $f$ admitir polinômio de Taylor de ordem $k$, $P$ coincide com tal polinômio.

Denotaremos tal polinômio por $j^{k}$ e o chamaremos de $j \underline{a}$ to $k$ da $f(e m 0)$.

Def.II-1-3: $\mathrm{f}:\left(\mathbb{R}^{\mathrm{n}} ; 0\right) \longrightarrow\left(\mathbb{R}^{\mathrm{m}} ; 0\right)$

Diremos que o germe $f$ admite $j$ ato punctual de ordem $k$ na origem se cada coordenada

$f_{i}:\left(\mathbb{R}^{n} ; 0\right) \longrightarrow(\mathbb{R} ; 0) \quad, \quad 1 \leq i \leq m$,

de $f$ admite jato punctual de ordem $k$ na origem.

Em tal caso, chamaremos de jato k da $f($ em 0$)$ e denotaremos por $j^{k_{f}}$ à m-upla de polinômios $\left(j^{k_{1}}, \ldots, j k_{f_{m}}\right)$.

Notações:

$$
\begin{aligned}
& G^{k}(n, m)=\left\{f:\left(\mathbb{R}^{n} ; 0\right) \longrightarrow\left(\mathbb{R}^{m} ; 0\right) \mid f \text { admite jato } k\right\} \\
& G^{k}(n)=G^{k}(n, 1)
\end{aligned}
$$

Segue imediatamente que $G^{k+1}(n, m) \subset G^{k}(n, m)$. 


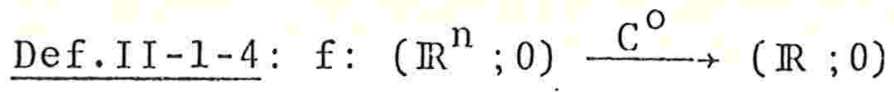

Diremos que a origem é ponto crítico de $f$ se $f \in G^{1}(n)$ e $\quad j^{1} f=0$.

II - 2 A DERIVADA RADIAL

Def.II-2-1: $U \subset \mathbb{R}^{\mathrm{n}}$ aberto, $0 \in \mathrm{U}$ ponto crítico de

$\mathrm{f:U} \stackrel{\mathrm{C}^{1}}{\longrightarrow} \mathbb{R}$

Chamaremos de derivada radial de $f$ e denotaremos por $f^{\prime}$ à função

$f^{\prime}: U \stackrel{\mathrm{C}^{\circ}}{\longrightarrow} \mathbb{R}$

$x \longmapsto<\operatorname{grad} f(x)|x\rangle$

Def.II-2-2: $f:\left(\mathbb{R}^{\mathrm{n}} ; 0\right) \stackrel{\mathrm{C}^{1}}{\longrightarrow}(\mathbb{R} ; 0)$

Chamaremos de derivada radial do germe $f$ (ou ape nas de derivada radial de f) e denotaremos por $f^{\prime}$ ao germe de funções $C^{O}$ na origem $f^{\prime}:\left(\mathbb{R}^{n} ; 0\right) \longrightarrow(\mathbb{R} ; 0)$ cujos representantes são as derivadas radiais dos representantes do germe $f$.

Para obtermos os resultados que nos levarão a uma classificação dos pares $\left(f, f^{\prime}\right)$ relativamente aos pares de pontos críticos associados, enunciaremos inicialmente um lema que será utilizado ao longo de todo o capítulo. 
Lema II-2-1: Seja $f: U \stackrel{C^{1}}{\rightarrow} \mathbb{R}$, onde $U \subset \mathbb{R}^{n}$ é una vizinhança aberta da origem e $f(0)=0$.

Se para algum $\varepsilon>0$ tal que $B_{\varepsilon} c U$ existe $x_{\varepsilon} \epsilon_{\varepsilon} B_{\varepsilon}^{\prime}$ sa tisfazendo $f\left(x_{\varepsilon}\right)>0$ (respect. $f\left(x_{\varepsilon}\right)<0, f\left(x_{\varepsilon}\right)=0$ ) então existe $\tilde{x}_{\varepsilon} \in B_{\varepsilon}^{\prime}$ tal que $f^{\prime}\left(\tilde{x}_{\varepsilon}\right)>0$ (respect. $\left.f^{\prime}\left(\tilde{x}_{\varepsilon}\right)<0, f^{\prime}\left(\tilde{x}_{\varepsilon}\right)=0\right)$.

8 A função

$\mathfrak{f}:[0,1] \longrightarrow \mathbb{R}$

$t \longmapsto f(t)=f\left(t x_{\varepsilon}\right)$

estả bem definida, è $\mathrm{C}^{1}$ e satisfaz

$f(0)=0 \quad, f(1)=f\left(x_{\varepsilon}\right)$

Logo pelo Teorema do Valor Médio,

$f\left(x_{\varepsilon}\right)=f(1)-\mathfrak{f}(0)=\frac{d \mathfrak{f}(\bar{t})}{d t}$ para algum $\bar{t}_{\epsilon}(0,1)$.

Como

$f^{\prime}\left(\operatorname{tx}_{\varepsilon}\right)=\left\langle\operatorname{gradf}\left(\mathrm{tx}_{\varepsilon}\right) \mid t x_{\varepsilon}\right\rangle=t<\operatorname{gradf}\left(\operatorname{tx}_{\varepsilon}\right)\left|\mathrm{x}_{\varepsilon}\right\rangle=t \frac{\mathrm{df}}{\mathrm{dt}}(\mathrm{t})$ temos

$$
f^{\prime}\left(\bar{t} x_{\varepsilon}\right)=\bar{t} f\left(x_{\varepsilon}\right),
$$

donde segue a tese para $\tilde{x}_{\varepsilon}=\bar{t}_{\varepsilon}$

Observemos que na demonstração anterior, tudo o que fí zemos foi parametrizar o segmento de reta $\Gamma$ que 1 iga a origem do $\mathbb{R}^{\mathrm{n}}$ a $x_{\varepsilon}$, restringir $f$ a esse segmento e usar o Teorema do Valor Médio.

E importante notar que não foi relevante a parametriza 
ção de $r$ escolhida. Foi natural, neste caso, a parametrização escolhida, mas refaremos abaixo a demonstração do . Iema anterior usando uma outra parametrização que se mostrará mais sugestiva no capitulo III, quando enunciaremos um resultado mais geral.

8 Seja $r$ o segmento de reta de extremidades $x_{\varepsilon}$ e $0 \in \mathbb{R}^{n}$. parametrizemos $\Gamma-\{0\}$ por

$$
\begin{aligned}
x:(-\infty, 0] & \longrightarrow \mathbb{R}^{X} \\
t & \longrightarrow x(t)=e^{t I} x_{\varepsilon}
\end{aligned}
$$

onde $I \in M_{n}(\mathbb{R})$ é a matriz identidade.

A função

$$
\begin{aligned}
f:(-\infty, 0] & \longrightarrow \mathbb{R} \\
t & \longrightarrow f \circ x(t)
\end{aligned}
$$

fica bem definida, pois $\Gamma \subset B_{\varepsilon} c U$, e é $C^{1}$.

$$
\text { Como } f(0)=f\left(x_{\varepsilon}\right) \text { e } \lim _{t+-\infty} f(t)=0 \text {, existe } \bar{t}(-\infty, 0) \text { tal }
$$
que $\frac{d f(\bar{t})}{d t}$ tem mesmo sinal que $f\left(x_{\varepsilon}\right)$.

Por outro lado,

$$
\begin{aligned}
\frac{d f}{d t}(\bar{t}) & =\langle\operatorname{gradf}(x(\bar{t})) \mid \dot{x}(\bar{t})\rangle= \\
& =\langle\operatorname{gradf}(x(\bar{t})) \mid x(\bar{t})\rangle=f^{\prime}(x(\bar{t}))
\end{aligned}
$$

Portanto, tomando-se $\tilde{x}_{\varepsilon}=x(\bar{t})$ segue a tese.

para $f: U \stackrel{C^{1}}{\longrightarrow} \mathbb{R}$, onde $U c \mathbb{R}^{n} \vec{e}$ uma vizinhança aberta da origem, temos

$$
V^{\prime}(f)=\left\{x \in U \mid f^{\prime}(x)=0\right\}
$$


Em vista disto, a proposição I-4-3 è um corolärio do resultado abaixo, que por sua vez é consequềncia imediata do 1 ema II $-2-1$.

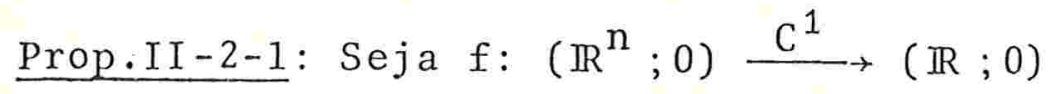

Se $f^{\prime}$ tem extremo forte na origem, então $f$ tam bëm tem extremo forte na origem, e este é do mesmo tipo que o de $f^{\prime}$.

\section{II-3 PROPRIEDADES DE $f^{\prime}$}

A proposição II-2-1 nos informa que se $f^{\prime}$ tem mäximo forte (respect. mínimo forte) na origem, o mesmo ocorre com f. Em vista disto surgiu naturalmente interesse por uma recíproca desta proposição.

A pergunta nos surgiu originariamente sob a forma: "Se $f:\left(\mathbb{R}^{n} ; 0\right) \stackrel{C^{1}}{\longrightarrow}(\mathbb{R} ; 0)$ tiver extremo forte na origem, pode mos concluir que $f^{\prime}$ também terä extremo forte na origem?".

A resposta a essa pergunta inicialmente nos surpreen-deu, por ser negativa mesmo no mundo dos polinômios.

Exemplo I I-3-1:

$$
\begin{aligned}
P(x, y) & =36 x^{2}-48 x y^{2}+17 y^{4} \\
& =\left(6 x-4 y^{2}\right)^{2}+y^{4} \text { tem mínimo forte na origem } \\
P^{\prime}(x, y) & =72 x^{2}-144 x y^{2}+68 y^{4} \\
& =72\left(x-y^{2}\right)^{2}-4 y^{4} \quad \text { tem sela na origem }
\end{aligned}
$$


No caso de polinômios de uma variável, P e p' tem sempre o mesmo tipo de extremo, como è simples observar.

As proposições a seguir têm como objetivo caracterizar os pares $\left(f, f^{\prime}\right)$ relativamente aos correspondentes pares de pontos críticos associados.

$$
f:\left(\mathbb{R}^{\mathrm{n}} ; 0\right) \stackrel{\mathrm{C}^{1}}{\rightarrow}(\mathbb{R} ; 0)
$$

Prop.II-3-1: Se f tem mínimo forte (respect. máximo forte) na origem, então a origem é ponto de mínimo forte, mínimo brando ou sela (respect. máximo forte, máximo brando ou sela) para $f^{\prime}$, e efeti vamente cada una dessas possibilidades se concretiza.

8 Se $f$ tem mínimo forte na origem, segue do Lema II-2-1 que existe uma sequência $\left\{x_{k}\right\}_{k \in \mathbb{N}}$ em $\mathbb{R}^{n}-\{0\}$ convergindo pa ra a origem tal que $f^{\prime}\left(x_{k}\right)>0$ para $k$ suficientemente grande. Logo, f' não pode ter máximo na origem.

Os exemplos II-3-1, II-3-1a, II-3-2, II-3-2a e II-3-3 mostram que efetivamente cada uma das possibilidades mencio nadas se concretiza.

Analogamente conclui-se o resultado respectivo caso $f$ tenha máximo forte na origem.

Exemplo II -3-1a:

Consideremos uma função $f: \mathbb{R} \stackrel{C^{1}}{\longrightarrow} \mathbb{R}$ com mínimo iso- 
1ado forte na origem e tal que a origem seja ponto de acumu lação de pontos crîticos de inflexão.

$$
\text { Neste caso, } f^{\prime}(x)=x \frac{d f}{d x}(x) \text { tem mínimo brando. }
$$

Exemp1o II-3-2:

$$
\begin{aligned}
P(x, y) & =36 x^{2}-48 x y^{2}+18 y^{4} \\
& =\left(6 x-4 y^{2}\right)^{2}+2 y^{4} \text { tem mínimo forte na origem } \\
P^{\prime}(x, y) & =72\left(x-y^{2}\right)^{2} \quad \text { tem mínimo brando na origem }
\end{aligned}
$$

Exemp1o II-3-2a:

Seja $f: \mathbb{R} \stackrel{\mathrm{C}^{1}}{\longrightarrow} \mathbb{R}$ com mínimo forte na origem, tal que a origem não seja ponto de mínimo local isolado.

$$
\text { Então } f^{\prime}(x)=x \frac{d f}{d x}(x) \text { tem sela na origem. }
$$

Exemp1o II -3-3:

$$
\begin{array}{ll}
P(x, y)=x^{2}+y^{2} & \text { tem mínimo forte na origem } \\
P^{\prime}(x, y)=2\left(x^{2}+y^{2}\right) & \text { tem mínimo forte na origem }
\end{array}
$$

Prop.II-3-2: Se f tem mínimo brando (respect. máximo brando) na origem, então a origem è ponto de mínimo brando ou sela (respect. máximo brando ou sela) para $f^{\prime}$, e cada uma dessas possibilidades de fato ocorre.

8 Se $f$ tem minimo brando na origem, segue do Lema II-2-1 que existem sequências $\left\{x_{k}\right\}_{k \in \mathbb{N}}$ e $\left\{y_{k}\right\}_{k \in \mathbb{N}}$ em $\mathbb{R}^{n}-\{0\}$, am 
bas convergentes para a origem, tais que $f^{\prime}\left(x_{k}\right)>0$ e $f^{\prime}\left(y_{k}\right)=0$ para $k$ suficientemente grande.

Logo, $f^{\prime}$ não pode ter nem mínimo forte nem máximo na origem.

Caso $f$ tenha máximo brando na origem, um raciocínio análogo leva ao resultado respectivo.

Os exemplos II-3-4, II-3-4a, II-3-5 e II-3-5a comple-tam a prova.

Exemp1o II-3-4:

$$
\begin{array}{ll}
P(x, y)=x^{2} & \text { tem mínimo brando na origem } \\
P^{\prime}(x, y)=2 x^{2} & \text { tem mínimo brando na origem }
\end{array}
$$

Exemplo II $-3-4 a$;

$$
\begin{array}{ll}
f(x)= \begin{cases}0 & , x \leq 0 \\
x^{2} & , x>0\end{cases} \\
f^{\prime}(x)=\left\{\begin{array}{ll}
0, & x \leq 0 \\
2 x^{2} & , x>0
\end{array}\right. \text { tem mínimo brando na origem }
\end{array}
$$

Exemp10 II-3-5:

$$
\begin{aligned}
P(x ; y) & =x^{2}-2 x y^{2}+y^{4} \\
& =\left(x-y^{2}\right)^{2} \quad \text { tem mínimo brando na origem } \\
P^{\prime}(x, y) & =2 x^{2}-6 x y^{2}+4 y^{4} \\
& =2\left(x-\frac{3}{2} y^{2}\right)^{2}-\frac{1}{2} y^{4} \quad \text { tem sela na origem }
\end{aligned}
$$


Exemp1o II-3-5a:

$$
\begin{array}{lr}
f(x)=x^{4} \operatorname{sen}^{2} \frac{1}{x} & \text { tem mínimo brando na origem } \\
f^{\prime}(x)=2 x^{3}\left(2 x \operatorname{sen} \frac{1}{x}-\cos \frac{1}{x}\right) \operatorname{sen} \frac{1}{x} \quad \text { tem sela na origem }
\end{array}
$$

Prop.II-3-3: Se $f$ tem sela na origem, então $f^{\prime}$ tem sela na origem.

8 Segue do Lèma II-2-1 que existem sequências $\left\{x_{k}\right\}_{k \in \mathbb{N}}$ e $\left\{\mathrm{y}_{\mathrm{k}}\right\}_{\mathrm{k} \in \mathbb{N}}$ em $\mathbb{R}^{\mathrm{n}}-\{0\}$, ambas convergentes para a origem, tais que $f^{\prime}\left(x_{k}\right)>0$ e $f^{\prime}\left(y_{k}\right)<0$ para todo $k$ suficientemen te grande.

Prop.II-3-4:Se $f$ tem ponto crítico na origem, então $f^{\prime}$ tem ponto crítico na origem.

$8 \mathrm{f}$ è $\mathrm{C}^{1}$, portanto gradf $\overrightarrow{\mathrm{e}} \mathrm{C}^{\mathrm{O}}$, e como a origem è ponto crítico de $f$ temos $\operatorname{gradf}(0)=0 \in \mathbb{R}^{\mathrm{n}}$.

$$
\text { Como } \left.\frac{\left|f^{\prime}(x)\right|}{|x|}=|<\operatorname{gradf}(x)| \frac{x}{x}\right\rangle|\leq| \operatorname{gradf}(x) \mid \quad \forall x \neq 0
$$

segue que

$$
\begin{aligned}
& \lim _{x \rightarrow 0} \frac{f^{\prime}(x)}{|x|}=0 \\
& \log 0, \quad f^{\prime} \in G^{1}(n) \quad \text { e } \quad j^{1} f^{\prime}=0
\end{aligned}
$$


II - 4 CLASS IFICAČ̃O DOS PARES $\left(f, f^{\prime}\right)$ PARA $f:\left(\mathbb{R}^{n} ; 0\right) \stackrel{C^{1}}{\longrightarrow}(\mathbb{R} ; 0)$

\begin{tabular}{|c|c|c|}
\hline $\begin{array}{l}\text { Singularidade de } \\
\text { f na origem }\end{array}$ & $\begin{array}{l}\text { Singularidade de } \\
f^{\prime} \text { na origem }\end{array}$ & Exemplo $(n \geq 2)$ \\
\hline Sela & Sela & $f(x, y)=x^{2}-y^{2}$ \\
\hline Minino forte & $\begin{array}{l}\text { Mínimo forte } \\
\text { Mínimo brando } \\
\text { Sela }\end{array}$ & $\begin{array}{l}f(x, y)=x^{2}+y^{2} \\
f(x, y)=\left(6 x-4 y^{2}\right)^{2}+2 y^{4} \\
f(x, y)=\left(6 x-4 y^{2}\right)^{2}+y^{4}\end{array}$ \\
\hline Mínimo brando & $\begin{array}{l}\text { Mínimo brando } \\
\text { Sela }\end{array}$ & $\begin{array}{l}f(x, y)=x^{2} \\
f(x, y)=\left(x-y^{2}\right)^{2}\end{array}$ \\
\hline Máximo brando & $\begin{array}{l}\text { Máximo brando } \\
\text { Sela }\end{array}$ & \\
\hline Máximo forte & $\begin{array}{l}\text { Mäximo forte } \\
\text { Máximo brando } \\
\text { Sela }\end{array}$ & . \\
\hline e $n$ & existem outras & ssibilidades \\
\hline
\end{tabular}




\section{II - 5 UMA CLASSE EM QUE. OS PARES ( $\left.f, f^{\prime}\right)$ SÃO TRIVIAIS}

Estamos interessados, neste parägrafo, em determinar uma classe de funções $C^{1}$ para a qual $f$ e $f^{\prime}$ tenham o mesmo tipo de ponto crítico. Já sabemos que na classe dos polinômios a uma variảve1 isto ocorre. Tentaremos captar a essência desse fenômeno nessa classe, para podermos chegar a uma classe mais ampla.

Dado um polinômio P: $(\mathbb{R} ; 0) \longrightarrow(\mathbb{R} ; 0)$ não identica-mente nulo, o monômio de menor grau de $\mathrm{P}$ determina seu comportamento na origem. Como o monômio de menor grau de $\mathrm{P}^{\prime}$ é, a menos de constante multiplicativa positiva, igual ao de P, segue que $\mathrm{P}^{\prime}$ tem mesmo tipo de comportamento na origem que P.

Essencialmente, essa é a idéia que nos permitirá am-pliar a classe em que $f$ e $f^{\prime}$ têm mesmo tipo de ponto crítico.

E imediato o seguinte resultado:

Prop.II-5-1 $: f:\left(\mathbb{R}^{\mathrm{n}} ; 0\right) \longrightarrow\left(\mathbb{R}^{\mathrm{m}} ; 0\right)$

$f$ tem jato punctual de ordem $k$ na origem se e somente se existem polinômios

$\mathrm{P}_{\mathrm{j}}: \mathbb{R}^{\mathrm{n}} \longrightarrow \mathbb{R} \quad, \quad 1 \leq j \leq m$

de grau menor ou igual a $k$, tais que

$$
\lim _{x \rightarrow 0} \frac{\left|f(x)-\left(P_{1}(x), \ldots, P_{m}(x)\right)\right|}{|x|^{k}}=0
$$


Observemos agora que para $f:\left(\mathbb{R}^{n} ; 0\right) \stackrel{C^{1}}{\longrightarrow}(\mathbb{R} ; 0)$ com ponto crítico na origem, se $f \in G^{k}(n)$ e $\operatorname{grad} f \in G^{k-1}(n, n)$, então

$\operatorname{grad} \cdot j^{k}=j^{k-1} \operatorname{grad} f$

E imprescindíve1, para o resultado acima, que $\operatorname{grad} f \in \mathrm{G}^{\mathrm{k}-1}(n, n)$.

o exemplo seguinte mostra que isso não é decorrência das outras hipóteses, e servirā para motivar a colocação das próximas proposições.

Exemp1o II-5-1:

$$
\begin{aligned}
& f:(\mathbb{R} ; 0) \stackrel{C^{1}}{\longrightarrow}(\mathbb{R} ; 0) \\
& f(x)=x^{3} \operatorname{sen} \frac{1}{x} \\
& f \in G^{2}(1), j^{2} f=0 \\
& \frac{d f}{d x}(x)=3 x^{2} \text { sen } \frac{1}{x}-x \cos \frac{1}{x} \notin G^{1}(1,1)=G^{1}(1)
\end{aligned}
$$

Prop.II-5-2: Seja $F:\left(\mathbb{R}^{\mathrm{n}} ; 0\right) \stackrel{\mathrm{C}^{\mathrm{O}}}{\longrightarrow}\left(\mathbb{R}^{\mathrm{n}} ; 0\right), F \in \mathrm{G}^{\mathrm{k}-1}(\mathrm{n}, \mathrm{n})$,

$$
\begin{aligned}
& j^{k-1} F=0 \text {. Então } \\
& g:\left(\mathbb{R}^{n} ; 0\right) \longrightarrow(\mathbb{R} ; 0)
\end{aligned}
$$

definida por

$$
\begin{aligned}
& g(x)=\int_{0}^{1}\langle F(t x) \mid t x\rangle d t \\
& \text { è um elemento de } G^{k}(n) \text { e } j^{k} g=0
\end{aligned}
$$




$$
\begin{aligned}
8 \quad \frac{g(x)}{|x|^{k}} & =\int_{C}^{1} \frac{t^{k}}{|t x|^{k}}\langle F(t x) \mid t x\rangle d t \\
& =\int_{0}^{1} t^{k}\left\langle\frac{F(t x)}{|t x|^{k-1}\left|\frac{t x}{|t x|}\right\rangle d t}\right.
\end{aligned}
$$

Portanto

$$
\begin{aligned}
\frac{|g(x)|}{|x|^{k}} & \leq \frac{1}{k+1} \sup _{t \in(0,1)} \frac{|F(t x)|}{|t x|^{k-1}} \\
& \leq \frac{1}{k+1} \sup _{y \in B_{|x|}} \frac{|F(y)|}{|y|^{k-1}}
\end{aligned}
$$

Como $j^{k-1} F=0$ temos $\lim _{x \rightarrow 0} \frac{|F(x)|}{|x|^{k-1}}=0$, donde

$$
\begin{aligned}
& \lim _{x \rightarrow 0} \sup _{y \in B} \frac{F(y)}{|y|^{k-1}}=0 \\
& \text { Logo, } \lim _{x \rightarrow 0} \frac{g(x)}{|x|^{k}}=0
\end{aligned}
$$

Corolärio II-5-2a $:$ Se $f:\left(\mathbb{R}^{n} ; 0\right) \stackrel{C^{1}}{\longrightarrow}(\mathbb{R} ; 0)$ é tal que

$$
\begin{aligned}
& \operatorname{grad} f \in G^{k-1}(n, n) \text { e } j^{k-1} \operatorname{grad} f=0 \text {, então } \\
& f \in G^{k}(n) \text { e } j^{k} f=0
\end{aligned}
$$

$8 \quad$ Basta tomar

$$
F(x)=\operatorname{gradf}(x)
$$

e observar que $g(x)=\int<F(t x t x>\mid d t=f(x)$ 


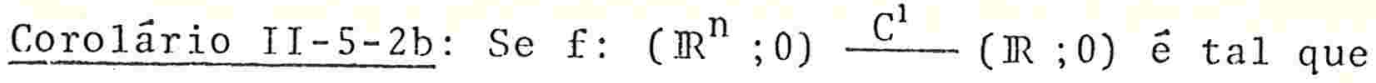
gradfe $G^{k-1}(n, n)$, então $f \in G^{k}(n) \quad e$ $\operatorname{grad} j^{k} f=j^{k-1} \operatorname{grad} f$

8 Basta tomar

$$
F(x)=\operatorname{gradf}(x)-j^{k-1} \operatorname{gradf}(x)
$$

e observar que

$$
\begin{aligned}
g(x) & =\int_{0}^{1}\langle\operatorname{grad} f(t x) \mid t x\rangle d t-\left\langle j^{k-1} \operatorname{gradf}(t x) \mid t x\right\rangle d t \\
& =f(x)-\int\left\langle j^{k-1} \operatorname{grad} f(t x) \mid t x\right\rangle d t .
\end{aligned}
$$

Então, como $j^{k-1} \operatorname{gradf}(t x)$ é uma $n$-upla de polinômios em $\left(t, x_{1}, \ldots, x_{n}\right)$, todos de grau menor ou igual a $k-1$ em $\left(x_{1}, \ldots, x_{n}\right)$, segue que

$$
P(x)=\int_{0}^{1}\left\langle j^{k-1} \operatorname{gradf}(t x) \mid t x\right\rangle d t
$$

é um polinômio de grau menor ou igual a $k$ em $\left(x, \ldots, x_{n}\right)$.

$$
\begin{aligned}
& \text { Logo, como } \\
& g=f-P \in G^{k}(n) \quad \text { e } \quad j^{k} g=0,
\end{aligned}
$$

segue que $f_{\epsilon} G^{k}(n)$ e $j^{k}=P$.

A observação que segue a proposição XI-5-1 completa a prova

Def.II-5-1: Dizemos que $\hat{\epsilon} \in \mathrm{G}^{\mathrm{r}}(\mathrm{n})$ ë $\underline{k \text {-decidirel }}(\mathrm{k} \leq \mathrm{r})$ se, a partir de $j^{k_{f}, e ́}$ possivel concluir que $0_{e} \mathbb{R}^{\text {nl }}$ é pon to de mínimo, mäximo ou sela de $f$. 
Denotemos por $A^{k}(n)$ o conjunto dos germes

$f:\left(\mathbb{R}^{\mathrm{n}} ; 0\right) \stackrel{\mathrm{C}^{1}}{ }(\mathbb{R} ; 0)$

com ponto crítico na origem, tais que $\operatorname{gradf} \varepsilon \mathrm{G}^{\mathrm{k}-1}(\mathrm{n}, \mathrm{n})$.

Pe1o corolärio II-5-2b, $A^{k}(n) \subset G^{k}(n)$.

Prop.II-5-3: Se $f \in A^{k}(n)$ é k-decidível e $j^{k-1} f=0$, então $f^{\prime} \in G^{k}(n), f^{\prime}$ e k-decidível e tem mesmo tipo de ponto crítico, na origem, que $f$.

$8 \quad f \in A^{k}(n) \subset G^{k}(n)$

$\Longrightarrow f=j^{k}+R \quad, \quad$ com $R \in A^{k}(n)$ e $j^{k} R=0$.

Portanto $f^{\prime}(x)=\langle\operatorname{grad} f(x) \mid x\rangle$

$$
=\left\langle\operatorname{gradj} \operatorname{gr}^{k}(x) \mid x\right\rangle+\langle\operatorname{gradR}(x) \mid x\rangle
$$

Como $j^{k-1} f=0$ temos que $j k_{f} \bar{e}$ um polinômio homogêneo de grau $k$, donde

$\left\langle\operatorname{grad} j^{k} f(x) \mid x\right\rangle=k j_{f}$ è um polinômio de grau $\leq k$ que é k-decidivel (pois $j^{k}$ é $k$-decidível).

$$
\begin{aligned}
& h(x)=\langle\operatorname{gradR}(x)| x>\in G^{k}(n) \text { e } j^{k} h=0 \quad \text {, pois } \\
& \frac{|h(x)|}{|2:|^{k}}=\mid\left\langle\frac{\operatorname{gradR}(x)}{|x|^{k-1}}\left|\frac{x}{|x|}\right| \leq \frac{|\operatorname{gradR}(x)|}{|x|^{k-1}}\right. \\
& j^{k-1} \operatorname{gradR}=\operatorname{grad} j^{k} R=0 . \\
& \text { Logo, } \\
& f^{\prime}=k j^{k} f+h \in G^{k}(n) \quad \text { e é k-decidivel. }
\end{aligned}
$$


Corolärio II-5-3a: Se $f \in A^{k}(1)$ e $j^{k-1} \frac{d f}{d x} \neq 0$, então $f^{\prime}$ tem mesmo tipo de ponto crítico que f.

8 Seja $s \leq k$ mínimo tal que $j^{s-1} \frac{d f}{d x} \neq 0$

Então $f \in A^{S}(1) \quad$ e $\quad \frac{d}{d x}\left(j^{S}: f\right)=j^{s-1} \frac{d f}{d x} \neq 0$

Logo, $j^{\mathrm{s}} f \neq 0$, donde $f$ è s-decidivel (pois é função de uma variảve1) .

Alêm disso, $\frac{d}{d x}\left(j^{s-1} f\right)=j^{s-2} \frac{d f}{d x}=0$, pela escolha de $s$. Logo, $j^{s-1} f \vec{e}$ constante, e como $f(0)=0, j^{s-1} f=0$.

Pela proposição anterior, $f^{\prime} \in G^{S}(1), f^{\prime}$ é s-decidível e tem mesmo tipo de ponto crítico, na origem, que $f$.

Exemp1o II-5-2:

Os polinômios a 1-variảvel com ponto crítico na origem não identicamente nulos satisfazem as hipóteses do corolário I I - 5-3a .

Exemp1o II-5-3:

Se $\mathrm{P}: \mathbb{R}^{\mathrm{n}} \longrightarrow \mathbb{R}$ è um polinômio não identicamente nu10 com ponto crítico na origem e o primeiro jato não nulo de $P$ é decidivel, então $P$ e $P^{\prime}$ têm o mesmo tipo de ponto crítico na origem. 
II-6 ESTUDO DE ALGUNS EXEMPLOS

Exemplo II-6-1:

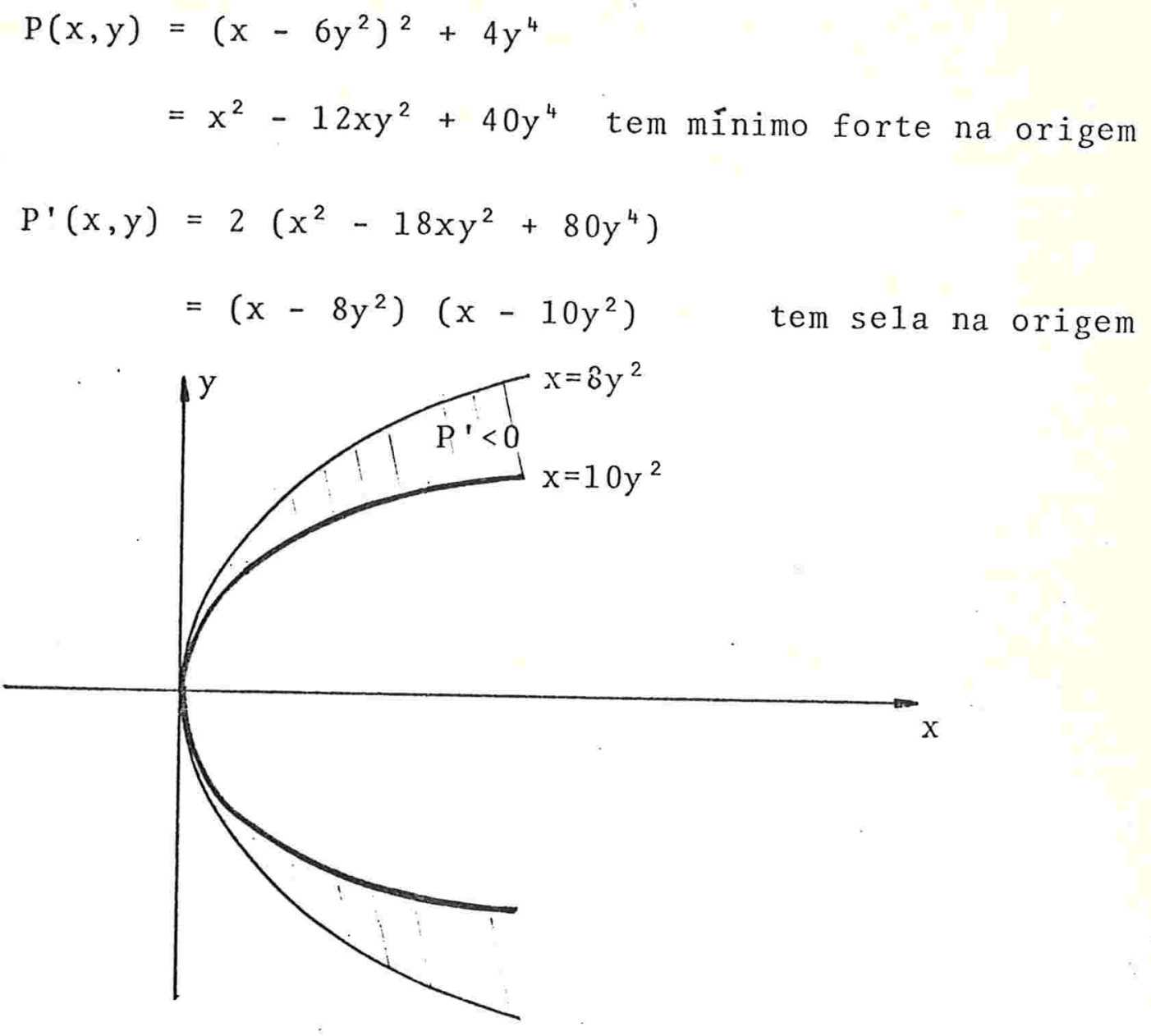

Fixemos $\mathrm{K}>0$

A curva de nivel $4 \mathrm{~K}^{4}$ de $\mathrm{P}$ fica definida por $x=6 y^{2} \pm 2 \sqrt{K^{4}-y^{4}} \quad, \quad|y| \leq K$

Estudemos as funções $x_{+} e x_{-}$definidas por $x_{ \pm}(y)=6 y^{2} \pm 2 \sqrt{K^{4}-y^{4}} \quad, \quad|y| \leq K$

Temos:

$x_{ \pm}^{\prime}(y)=4 y\left(3 \mp \frac{y^{2}}{\sqrt{K^{4}-y^{4}}}\right) \quad, \quad|y|<K$ 


$$
x_{ \pm}^{\prime \prime}(y)=12 \pm \frac{4 y^{2}\left(y^{4}-3 K^{4}\right)}{\left(\sqrt{K^{4}-y^{4}}\right)} \quad, \quad|y|<K
$$

Sinal de $x_{ \pm}$e $x_{ \pm}^{\prime}$

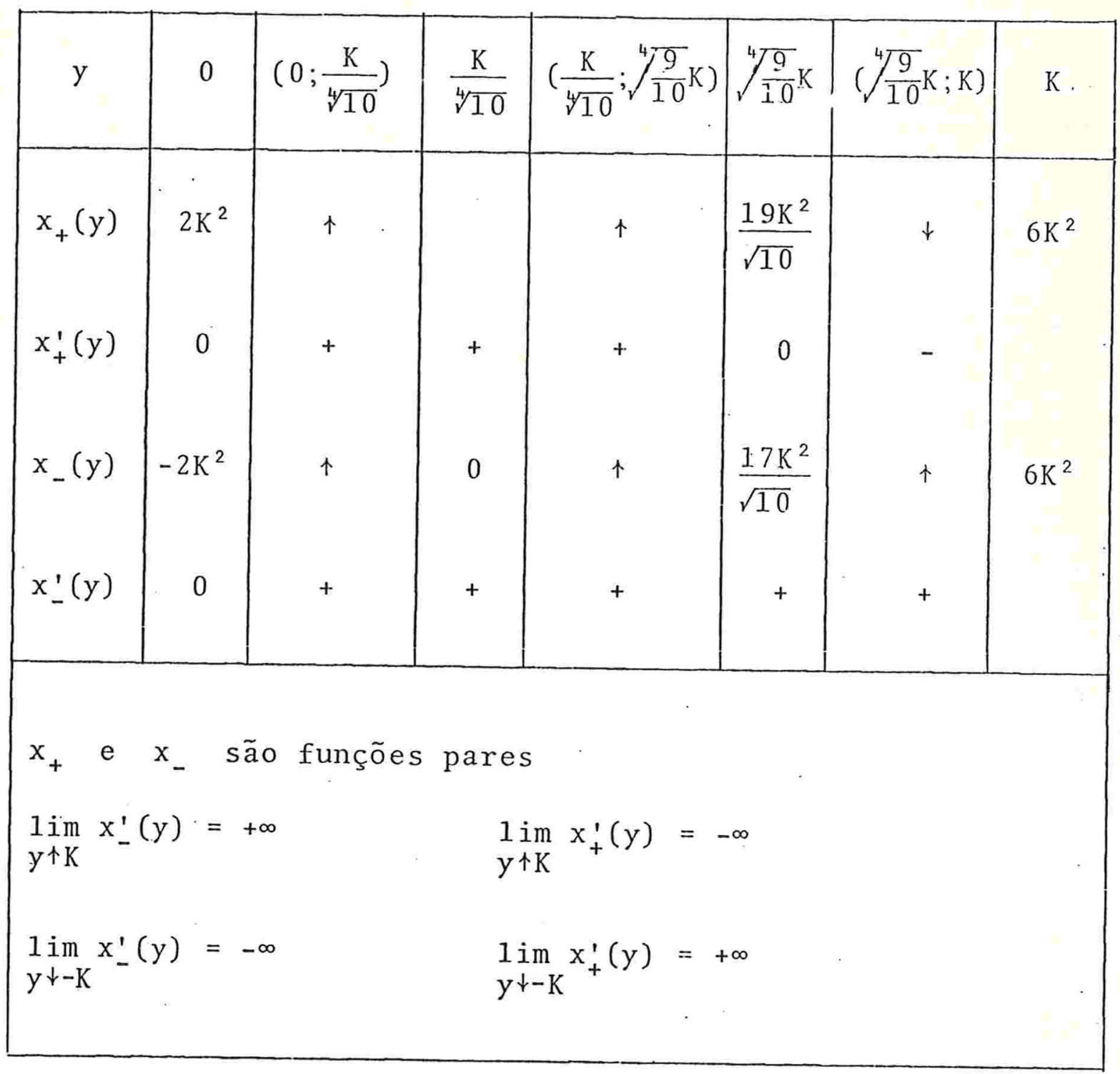


Concavidade de $x_{+}$e $x_{-}$

$x_{ \pm}^{\prime \prime}=12 \pm \frac{4 y^{2}\left(y^{4}-3 K^{4}\right)}{\left(\sqrt{K^{4}-y^{4}}\right)^{3}} \quad, \quad|y|<K$

Logo ,

$$
x_{-}^{\prime \prime}(y)>0 \quad, \quad \forall y \quad(|y|<K)
$$

Para $|y|<K$ temos

$x_{+}^{\prime \prime}(y)=0 \Longleftrightarrow 9 K^{12}-36 K^{8} y^{4}+33 K^{4} y^{8}-10 y^{12}=0$

Seja

$g(\lambda)=10 \lambda^{3}-33 K^{4} \lambda^{2}+36 K^{8} \lambda \quad, \quad \lambda \geq 0$

Observemos que

$x_{+}^{\prime \prime}(y)=0 \Longleftrightarrow g\left(y^{4}\right)=9 K^{12}$

Como $g^{\prime}(\lambda)=30\left(\lambda-\frac{6}{5} K^{4}\right)\left(\lambda-K^{4}\right), \quad \lambda \geq 0, \quad$ e

$g\left(\frac{6}{5} K^{4}\right)=12,96 K$

$g\left(K^{4}\right)=13 K^{12}$

$g(0)=0$

temos:

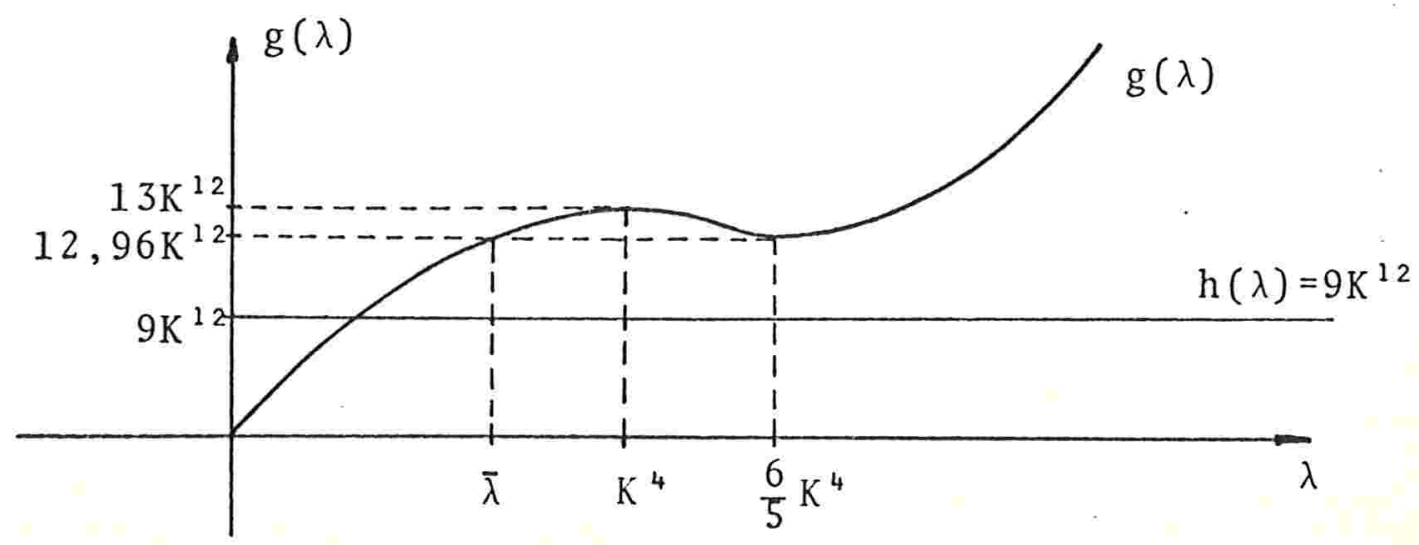


Logo, $\bar{\exists} ! \bar{\lambda} \epsilon\left(0, K^{4}\right)$ tal que $g(\bar{\lambda})=9 K^{12}$

Assim, $x_{*}^{\prime \prime}$ tem uma única raiz positiva $\bar{y} \epsilon(0, K)$, e conse-quentemente, $\bar{y}$ e $-\bar{y}$ são suas ünicas raízes $(e m(-K, K))$.

Como

$$
\left.x_{+}^{\prime \prime}\left(+\frac{4 / \overline{9}}{\sqrt{10}} K\right)<0 \quad \text { e } \quad x_{+}^{\prime \prime \prime}+\frac{K}{\sqrt[4]{10}}\right)>0
$$

segue que $\quad \bar{y} \in\left(\frac{K}{\sqrt[4]{10}}, \frac{4}{\sqrt{10}} K\right)$

Assim

$$
\begin{aligned}
& \exists \bar{y} \in\left(\frac{\mathrm{K}}{\sqrt[4]{10}}, \frac{\sqrt[4]{9}}{\sqrt{10}} \mathrm{~K}\right) \quad \text { tal que } \\
& x_{+}^{\prime \prime}(-\bar{y})=0 \\
& x_{+}^{\prime \prime}(y)>0 \quad \text { se } \quad-\bar{y}<y<\bar{y} \quad: \\
& x_{+}^{\prime \prime}(y)<0 \quad \text { se } \quad-K<y<-\bar{y} \quad \text { ou } \quad \bar{y}<y<K
\end{aligned}
$$


$-48-$

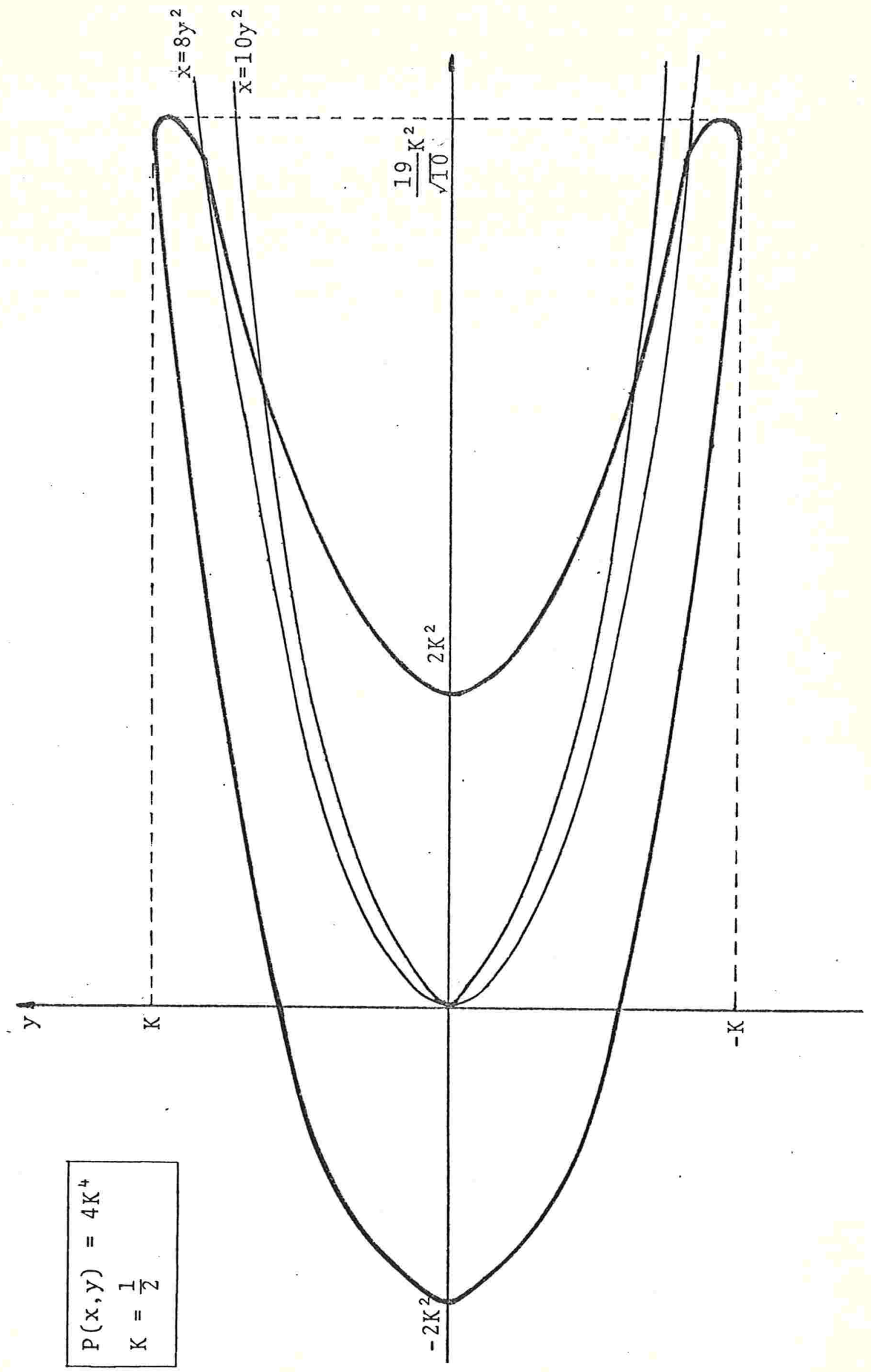


Exemp1o II-6-2:

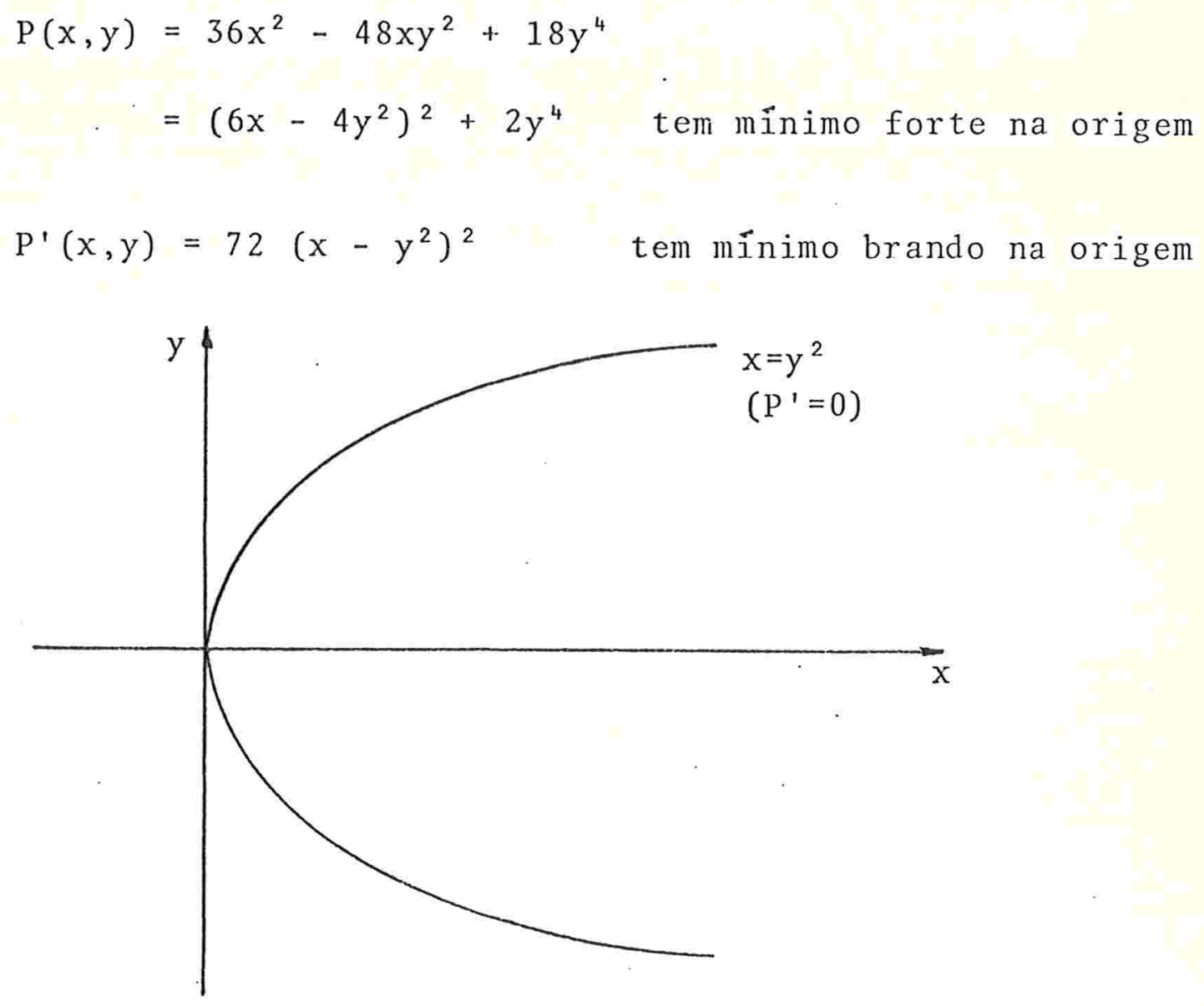

Fixemos $K>0$

A curva de nível $2 \mathrm{~K}^{4}$ de $\mathrm{P}$ fica definida por $x=\frac{4 y^{2} \pm \sqrt{2} \sqrt{K^{4}-y^{4}}}{6}$ , $\quad|y| \leq K$

Estudemos as funções $x_{ \pm}(y)=\frac{4 y^{2} \pm \sqrt{2} \sqrt{K^{4}-y^{4}}}{6}$ :

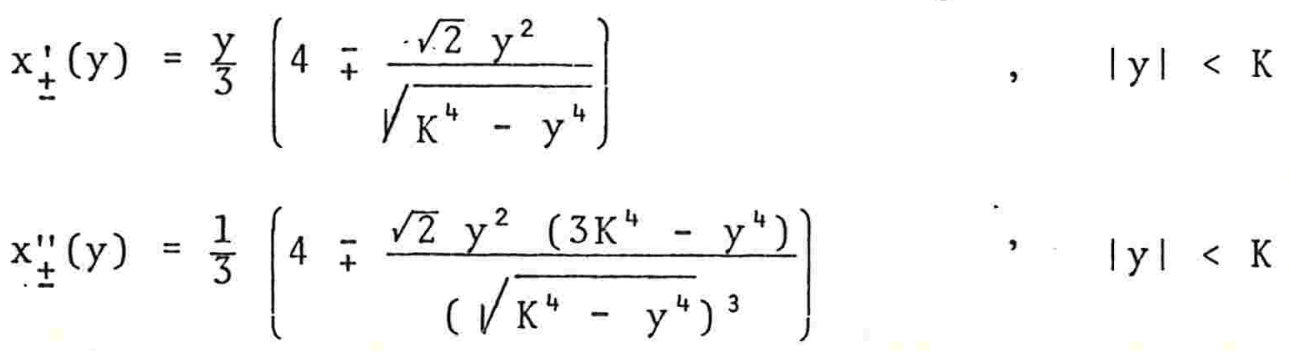


Sinal de $x_{ \pm}$e $x_{ \pm}^{\prime}$

\begin{tabular}{|c|c|c|c|c|c|c|c|}
\hline$y$ & 0 & $\left(0 ; \frac{K}{\sqrt[4]{8}}\right)$ & $\frac{K}{\sqrt[4]{8}}$ & $\left(\frac{K}{\sqrt{8}} ; V^{4 / \frac{8}{9}} K\right)$ & $y^{4 / \overline{8}} \mathrm{~K}$ & $\left(l^{4} / \overline{8} \overline{9} K ; K\right)$ & K \\
\hline$x_{+}(y)$ & $\frac{\sqrt{2}}{6} K^{2}$ & $\uparrow$ & & $\uparrow$ & $\frac{\sqrt{2}}{2} K^{2}$ & $\downarrow$ & $\frac{2}{3} K^{2}$ \\
\hline$x_{+}^{\prime}(y)$ & 0 & + & & + & 0 & - & \\
\hline$x_{-}(y)$ & $-\frac{\sqrt{2}}{6} K^{2}$ & $\uparrow$ & & $\uparrow$ & & $\uparrow$ & $\frac{2}{3} K^{2}$ \\
\hline$x_{-}^{\prime}(y)$ & 0 & + & & + & & + & \\
\hline$x_{+} \quad e$ & $x_{\text {_ }}$ são & funções & pares & & & & \\
\hline $\lim _{y \uparrow K} x_{-}^{\prime}$ & $(y)=+o$ & & $\begin{array}{l}1 \mathrm{im} \\
\mathrm{y} \uparrow \mathrm{K}\end{array}$ & $x_{+}^{\prime}(y)=-$ & & & \\
\hline $\lim _{y \downarrow-K} x_{-}^{\prime}$ & $(y)=-0$ & o & $\begin{array}{l}1 \mathrm{im} \\
y^{\downarrow}-K\end{array}$ & $x_{+}^{\prime}(y)=+$ & $\infty$ & : & \\
\hline
\end{tabular}

Concavidade de $x_{+}$e $x_{-}$

$$
\begin{aligned}
& x_{ \pm}^{\prime \prime}(y)=\frac{1}{3}\left(4 \mp \frac{\left.\sqrt{2} y^{2} \frac{\left(3 K^{4}-y^{4}\right)}{\left(\sqrt{K^{4}-y^{4}}\right)^{3}}\right)}{}(|y|<k\right. \\
& \text { Logo, } \quad x_{-}^{\prime \prime}(y)>0 \quad \forall y \quad(|y|<K)
\end{aligned}
$$


Para $|y|<K$ temos

$x_{+}^{\prime \prime}(y)=0 \Longleftrightarrow 3 y^{12}-10 K^{4} y^{8}+11 K^{8} y^{4}-\frac{8}{3} K^{12}=0$

Seja

$g(\lambda)=3 \lambda^{3}-10 K^{4} \lambda^{2}+11 K^{8} \lambda \quad, \quad \lambda \geq 0$

Temós:

$x_{+}^{\prime \prime}(y)=0 \Longleftrightarrow g\left(y^{4}\right)=\frac{8}{3} K^{12}$

Como

$$
\begin{aligned}
& g^{\prime}(\lambda)=9 \lambda^{2}-20 K^{4} \lambda+11 K^{8} \\
& =9\left(\lambda-K^{4}\right)\left(\lambda-\frac{11}{9} K^{4}\right) \quad, \quad \lambda \geq 0, \quad \text { e } \\
& g\left(\frac{11}{9} K^{4}\right)=\frac{968}{243} K^{12}>\frac{8}{3} K^{12} \\
& g\left(K^{4}\right)=4 K^{12}>\frac{8}{3} K^{12} \\
& g(0)=0
\end{aligned}
$$

temos

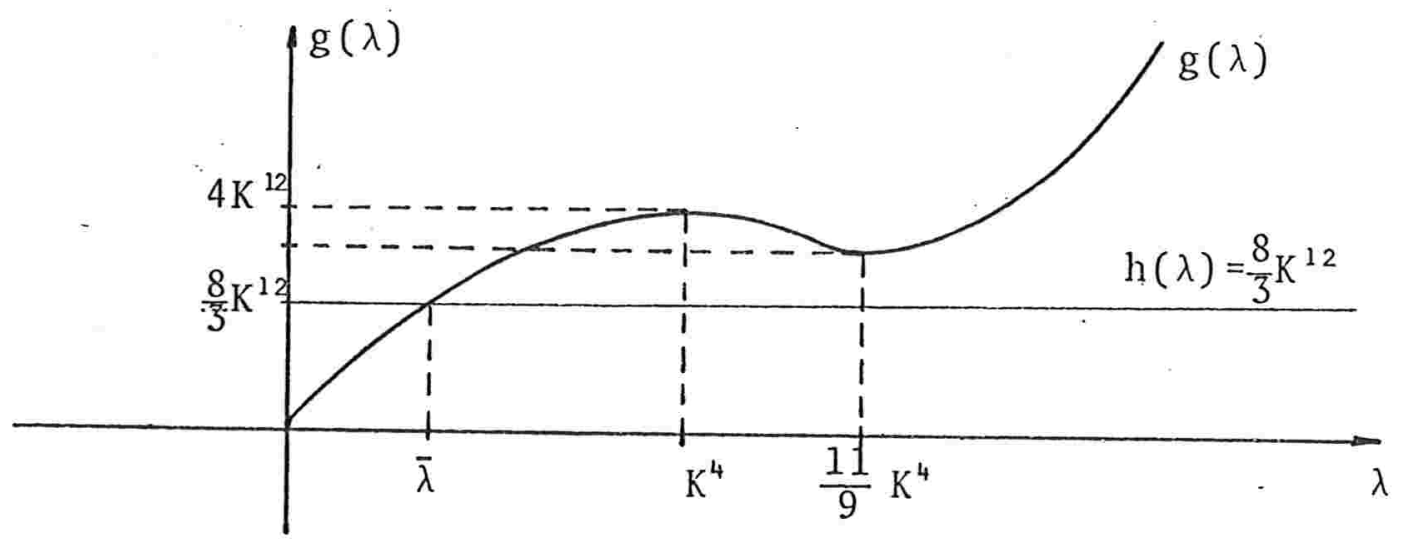

Logo, $\exists ! \quad \bar{\lambda} \in\left(0, K^{4}\right)$ tal que $g(\bar{\lambda})=\frac{8}{3} K^{12}$. 
Portanto, as raízes de $x_{+}^{\prime \prime}$ em $(-K, K)$ são exatamente $\pm \bar{y}$, onde $\bar{y}=\sqrt[4]{\bar{\lambda}}$

$$
\begin{aligned}
\text { Como } & x_{+}^{\prime \prime}\left(\sqrt[4]{\frac{1}{3}} K\right)=0 \\
\text { segue que } & \bar{y}=\sqrt[4]{\frac{1}{3}} K
\end{aligned}
$$

Assim:

$$
\begin{aligned}
& x_{+}^{\prime \prime}\left( \pm \sqrt[4]{\frac{1}{3}} \mathrm{~K}\right)=0 \\
& x_{+}^{\prime \prime}(\mathrm{y})>0 \text { se }-\sqrt[4]{\frac{1}{3}} \mathrm{~K}<\mathrm{y}<\sqrt[4]{\frac{1}{3}} \mathrm{~K} \\
& x_{+}^{\prime \prime}(\mathrm{y})<0 \text { se } \quad-\mathrm{K}<\mathrm{y}<-\sqrt[4]{\frac{1}{3}} \mathrm{~K} \text { ou } \sqrt[4]{\frac{1}{3}} \mathrm{k}<\mathrm{y}<\mathrm{K}
\end{aligned}
$$


$-53-$

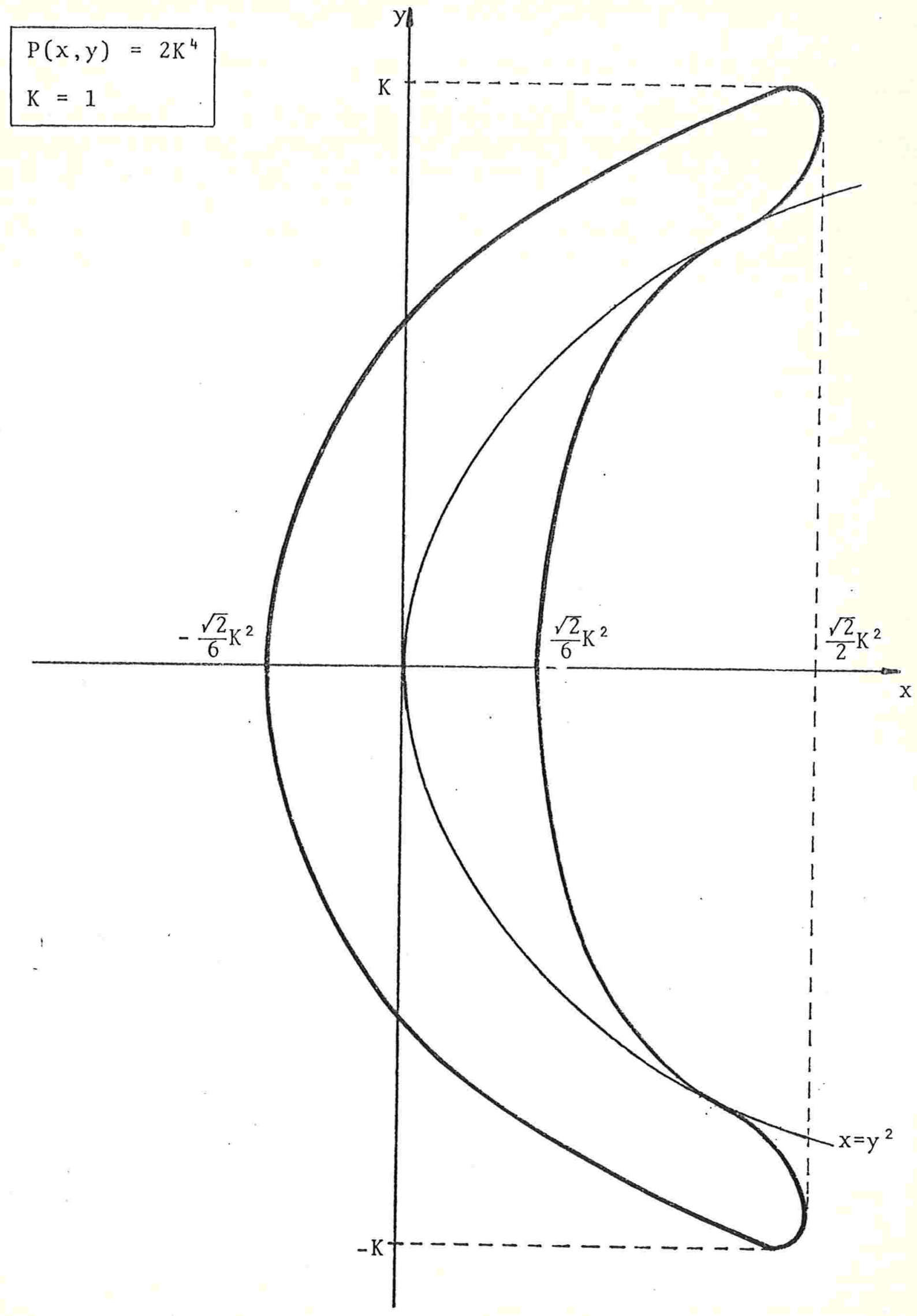


C A P I T U L O

A derivada radial relativa a um produto interno 


\section{I I - 1 I NTRODUÇÃO}

No capítulo anterior, a partir do produto interno usual do $\mathbb{R}^{\mathrm{n}}$, definimos canonicamente uma aplicação do espaço dos germes de funções $C^{1}$ na origem, no espaço dos germes de funções $C^{\circ}$ na origem, associando a cada $f:\left(\mathbb{R}^{n} ; 0\right) \stackrel{C^{1}}{\longrightarrow}(\mathbb{R} ; 0)$ sua derivada radial $f^{\prime}:\left(\mathbb{R}^{\mathrm{n}} ; 0\right) \stackrel{\mathrm{C}^{\mathrm{O}}}{\longrightarrow}(\mathbb{R} ; 0)$, e obtivemos algumas propriedades de tal aplicação.

Neste capítulo, faremos um estudo análogo tomando em $\mathbb{R}^{\mathrm{n}}$ um produto interno arbitrário.

\section{III-2 A DERI VADA "RADIAL" $\mathrm{f}_{\mathrm{A}}^{\prime}$}

No que segue, $A \in M_{n}(\mathbb{R})$ será uma matriz simëtrica, definida positiva.

Def.III-2-1: $U \subset \mathbb{R}^{\mathrm{n}}$ aberto, $0 \in U, f: U \stackrel{\mathrm{C}^{1}}{\longrightarrow} \mathbb{R}, f(0)=0$. Chamaremos de derivada radial de $f$ com respeito a A e denotaremos por $f_{A}^{\prime}$ à função

$$
\begin{aligned}
f_{A}^{\prime}: U \stackrel{C^{O}}{\longrightarrow} \mathbb{R} \\
\quad x \longmapsto f_{A}^{\prime}(x)=\langle\operatorname{gradf}(x) \mid A x\rangle
\end{aligned}
$$

$\underline{\text { Def.III-2-2 }}: \mathrm{f}:\left(\mathbb{R}^{\mathrm{n}} ; 0\right) \stackrel{\mathrm{C}^{1}}{\longrightarrow}(\mathbb{R} ; 0)$

Chamaremos de derivada radial de $f$ com respeito a A e denotaremos por $f_{A}^{\prime}$ ao germe de funções $C^{0}$ na origem $\mathrm{f}_{\mathrm{A}}^{\prime}:\left(\mathbb{R}^{\mathrm{n}} ; 0\right) \stackrel{\mathrm{C}^{\mathrm{O}}}{\longrightarrow}(\mathbb{R} ; 0)$ 
cujos representantes são as derivadas radiais com respeito a $A$ dos representantes de $f$.

$\underline{\text { Def.III-2-3 }}: \mathrm{f}: \mathbb{R}^{\mathrm{n}} \stackrel{\mathrm{C}^{1}}{\longrightarrow} \mathbb{R} \quad, \quad f(0)=0, \operatorname{grad} f(0)=0$ $V_{A}^{\perp}(f)=\left\{x \in \mathbb{R}^{n} \mid f_{A}^{\prime}(x)=0\right\}$

\section{I -3 EXEMPLOS}

$\mathrm{Na}$ maioria dos exemplos que apresentaremos neste parâgrafo, precisaremos de uma base $\left\{v_{1}, \ldots, v_{n}\right\}$ de $\mathbb{R}^{n}$ satisfa zendo certas propriedades, o que nos será fornecido pelo

Lema III-3-1: Seja $(V,<\mid \gg)$ um espaço vetorial real de dimensão finita, com produto interno.

Se $\quad \Psi: \mathrm{V} \longrightarrow \mathrm{V}$ é uma aplicação linear simé-trica, definida positiva, então todos os autovalores de $\psi$ são reais, positivos, e existe uma base ortonormal $\left\{v_{1}, \ldots, v_{n}\right\}$ de $V$ formada por autovetorés de $\psi$.

8 (a) Seja $\lambda \in \mathbb{C}$ um autovalor de $\psi$ e $v \in V$ um autovetor as sociado a $\lambda$.

Como $v \neq 0$ e $\psi$ é definida positiva, temos

$0<\langle\psi \mathrm{v} \mid \mathrm{v}\rangle=\lambda\langle\mathrm{v} \mid \mathrm{v}\rangle$

donde $\quad \lambda \in \mathbb{R} \quad$ e $\quad \lambda>0$

Is to prova a primeira afirmação da tese. 
(b) (Indução sobre dim V)

Se $\operatorname{dim} V=1$ nada hä a demonstrar.

Suponhamos a tese vâlida para espaços vetoriais com dimensão $n-1 \quad(n \geq 2)$.

Se $\operatorname{dim} V=n$, tomemos um subespaço vetorial $V$ de $V$, de dimensão $n-1$, e consideremos em $V$ o produto interno $\ll|\gg=\langle\mid\rangle|_{V_{1}} \times V_{1}$

Seja $\quad \tilde{\psi}=\psi / \mathrm{v}_{1}$

$\tilde{\psi}$ é uma aplicação linear simêtrica, definida positi va, e $\operatorname{dim} v_{1}=n-1$. Pela hipótese de indução, exis te uma base ortonormal (com respeito a «|»), $\left\{v_{1}, \ldots, v_{n-1}\right\}$ de $v_{1}$ formada por autovetores de $\tilde{\Psi}$ associados a autovalores $\lambda_{1}, \ldots, \lambda_{n}$.

Da definição de $\ll \mid \gg$ e de $\Psi$ segue imediatamente que $\left\{v_{1}, \ldots, v_{n-1}\right\}$ é um subconjunto ortonormal de $\mathrm{V}$ formado por autovetores de $\psi$ associados aos autovalores $\lambda_{1}, \ldots, \lambda_{n-1}$.

Completemos $\left\{v_{1}, \ldots, v_{n-1}\right\}$ a uma base ortonormal $\left\{v_{1}, \ldots, v_{n-1}, v_{n}\right\}$ de $v$.

Para $1 \leq j \leq n-1 \quad$ temos

$\left\langle\psi v_{n} \mid v_{j}\right\rangle=\left\langle v_{n} \mid \psi v_{j}\right\rangle=\lambda_{j}\left\langle v_{n} \mid v_{j}\right\rangle=0$

Logo, $\psi v_{n}=\lambda_{n} v_{n}$ para algum $\lambda_{n} \in \mathbb{R}$

Is to completa a prova. 
Corolário: Seja $A \in M_{n}(\mathbb{R})$ simétrica, definida positiva.

Existe uma base ortonorma1 $\left\{v_{1}, \ldots, v_{n}\right\}$ de $\mathbb{R}^{n}$ formada por autovetores de A, associados a autova lores positivos $\lambda_{1}, \ldots, \lambda_{n}$.

Fixemos $A \in M_{n}(\mathbb{R})$ simétrica, definida positiva.

Nos exemplos a seguir, $\left\{v_{1}, \ldots, v_{n}\right\}$ e $\lambda_{1}, \ldots, \lambda_{n}$ serão tomados como no corolário anterior.

Só apresentaremos exemplos para dimensão $n \geq 2$, pois no caso $n=1$ os exemplos apresentados no Cap.II se adaptam sem dificuldades ao contexto tratado aqui.

Exemp1o III-3-1:

$P\left(x_{1}, \ldots x_{n}\right)=P(x)=x_{1}^{2}-x_{2}^{2} \quad$ tem sela na origem

$P_{A}^{\prime}(x)=2<\left(x_{1},-x_{2}, 0, \ldots, 0\right) \mid A x>$

portanto

$$
\begin{array}{ll}
\mathrm{P}_{\mathrm{A}}^{\prime}\left(\mathrm{x}_{1} \mathrm{e}_{1}\right)=2 \mathrm{x}_{1}^{2}<\mathrm{e}_{1}\left|\mathrm{Ae}_{1}\right\rangle>0 & \forall \mathrm{x}_{1} \neq 0 \quad \mathrm{e} \\
\mathrm{P}_{\mathrm{A}}^{\prime}\left(\mathrm{x}_{2} \mathrm{e}_{2}\right)=-2 \mathrm{x}_{2}^{2}<\mathrm{e}_{2}\left|\mathrm{Ae}_{2}\right\rangle<0 & \forall \mathrm{x}_{2} \neq 0
\end{array}
$$

Logo ,

$\mathrm{P}_{\mathrm{A}}^{\prime}$ tem sela na origem 
Exemp1o III-3-2:

$$
P(x)=\left\langle x \mid v_{1}\right\rangle^{2} \text { tem mínimo brando na origem }
$$

$$
\begin{aligned}
P_{A}^{\prime}(x) & =\left\langle 2\left\langle x \mid v_{1}\right\rangle v_{1} \mid A x\right\rangle \\
& =2\left\langle x \mid v_{1}\right\rangle\left\langle v_{1} \mid A x\right\rangle \\
& =2 \lambda_{1}\left\langle x \mid v_{1}\right\rangle^{2}
\end{aligned}
$$

Logo ,

$$
\mathrm{P}_{\mathrm{A}}^{\prime} \text { tem mínimo brando na origem }
$$

Exemp1o III-3-3: Seja $m \in \mathbb{N}^{*}, \quad m>2 \frac{\lambda_{1}}{\lambda_{2}}$

$$
P(x)=\left(\left\langle x \mid v_{1}\right\rangle-\left\langle x \mid v_{2}\right\rangle^{m}\right)^{2} \text { tem minimo brando na origem }
$$

$$
\begin{aligned}
& P_{A}^{\prime}(x)=\left\langle 2\left(\left\langle x \mid v_{1}\right\rangle-\left\langle x \mid v_{2}\right\rangle^{m}\right)\left(v_{1}-m<x \mid v_{2}>^{m-1} v_{2}\right) \mid A x\right\rangle \\
& =2\left(\langle x| v_{1}>-\left\langle x \mid v_{2}\right\rangle^{m}\right)\left(\lambda_{1}<x\left|v_{1}>-m \lambda_{2}<x\right| v_{2}>^{m}\right)
\end{aligned}
$$

Portanto $P_{A}^{\prime}\left(t v_{1}\right)=2 \lambda_{1} t^{2}>0$ se $t \neq 0$, e $\mathrm{P}_{\mathrm{A}}^{\prime}\left(2 \mathrm{t}^{\mathrm{m}} \mathrm{v}_{1}+\mathrm{t} \mathrm{v}_{2}\right)=2 \mathrm{t}^{2 \mathrm{~m}}\left(2 \lambda_{1}-\mathrm{m}_{2}\right)<0$ se $\mathrm{t} \neq 0$ (pois $\left.\mathrm{m}>2 \frac{\lambda}{\lambda_{2}}\right)$. Logo ,

$P_{A}^{\prime}$ tem sela na origem 
Exemp1o III-3-4:

$$
P(x)=\sum_{j=1}^{n}\left\langle x \mid v_{j}\right\rangle^{2} \text { tem mínimo forte na origem }
$$

$$
\begin{aligned}
P_{A}^{\prime}(x) & =\left\langle 2 \sum_{j=1}^{n}\left\langle x \mid v_{j}\right\rangle v_{j} \mid A x\right\rangle \\
& =2 \sum_{j=1}^{n} \lambda_{j}\left\langle x \mid v_{j}\right\rangle^{2}
\end{aligned}
$$

Logo ,

$\mathrm{P}_{\mathrm{A}}^{\prime}$ tem mínimo forte na origem

Exemp1o III-3-5:

$$
\begin{aligned}
& \text { Sejam } m \in \mathbb{N}^{*}, \quad m \neq \frac{\lambda_{1}}{\lambda_{2}} \quad \text { e } a=\frac{\left(\lambda_{1}-m \lambda_{2}\right)^{2}}{4 m \lambda_{1} \lambda_{2}} \\
& Q(x)=\left(<x \mid v_{1}>-\left\langle x \mid v_{2}\right\rangle^{m}\right)^{2}+a<x\left|v_{2}\right\rangle^{2 m} \\
& R(x)=\sum_{j=3}^{n}\left\langle x \mid v_{j}\right\rangle^{2} \quad(\text { caso } n=2 \text { tomamos } R=0)
\end{aligned}
$$

$$
P(x)=Q(x)+R(x) \text { tem mínimo forte na origem (pois } a>0 \text { ) }
$$

$$
\begin{aligned}
& P_{A}^{\prime}(x)=Q_{A}^{\prime}(x)+R_{A}^{\prime}(x) \\
& R_{A}^{\prime}(x)=2 \sum_{\lambda=3}^{n} \lambda_{j}\left\langle x \mid v_{j}\right\rangle^{2} \geq 0 \text { e só se anula no subespaço }
\end{aligned}
$$

gerado por $\left\{v_{1}, v_{2}\right\}$. 


$$
\begin{array}{r}
Q_{A}^{\prime}(x)=2\left(\langle x| v_{1}>-\left\langle x \mid v_{2}\right\rangle^{m}\right)\left(\lambda_{1}<x\left|v_{1}>-m \lambda_{2}<x\right| v_{2}>^{m}\right)+ \\
+2 a m \lambda_{2}<x \mid v_{2} \\
=2\left[\left(\sqrt{\lambda_{1}}<x\left|v_{1}>-\frac{\left(\lambda_{1}+m \lambda_{2}\right)}{2 \sqrt{\lambda_{1}}}<x\right| v_{2}>^{m}\right)^{2}+\right. \\
\left.+\left(m(a+1) \lambda_{2}-\frac{\left(\lambda_{1}+m \lambda_{2}\right)^{2}}{4 \lambda_{1}}\right)<x \mid v_{2}>^{2 m}\right]
\end{array}
$$

Como $\quad a=\frac{\left(\lambda_{1}-m \lambda_{2}\right)^{2}}{4 m \lambda_{1} \lambda_{2}}$

$Q_{A}^{\prime}(x)=2\left(\sqrt{\lambda_{1}}<x\left|v_{1}>-\frac{\left(\lambda_{1}+m \lambda_{2}\right)}{2 \sqrt{\lambda_{1}}}<x\right| v_{2}>^{m}\right)^{2}$

Portanto $Q_{A}^{\prime}$ tem mínimo brando na origem e ainda tem mínimo brando quando restrito ao subespaço vetorial gerado por $\left\{v_{1}, v_{2}\right\}$.

Logo ,

$$
P_{A}^{\prime}=Q_{A}^{\prime}+R_{A}^{\prime} \quad \text { tem mínimo brando na origem }
$$

Exemp1o III-3-6:

Sejam $m \in \mathbb{N}^{*}, \quad m \neq \frac{\lambda_{1}}{\lambda_{2}}$ e $a \in \mathbb{R}$ tal que $0<a<\frac{\left(\lambda_{1}-m \lambda_{2}\right)^{2}}{4 m \lambda_{1} \lambda_{2}}, \quad$ e

$Q$ e R como no exemplo III-3-5.

$$
P(x)=Q(x)+R(x) \text { tem mínimo forte na origem }
$$


Já vimos no exemplo III-3-5 que $R_{A}^{\prime}(x) \geq 0$ e se anula exa tamente no subespaço vetorial gerado por $\left\{v_{1}, v_{2}\right\}$ e que

$$
\begin{aligned}
Q_{A}^{\prime}(x)=2\left[\left(\sqrt{\lambda_{1}}<x\left|v_{1}>-\frac{\left(\lambda_{1}+m \lambda_{2}\right)}{2 \sqrt{\lambda_{1}}}<x\right| v_{2}\right\rangle^{m}\right. & )^{2} \\
& \left.+\left(m(a+1) \lambda_{2}-\frac{\left(\lambda_{1}+m \lambda_{2}\right)^{2}}{4 \lambda_{1}}\right)<x \mid v_{2}>^{2 m}\right]
\end{aligned}
$$

Como $a<\frac{\left(\lambda_{1}-m \lambda_{2}\right)^{2}}{4 m \lambda_{1} \lambda_{2}}$ temos $m(a+1) \lambda_{2}-\frac{\left(\lambda_{1}+m \lambda_{2}\right)}{4 \lambda_{1}}<0$

Assim, Q' terá sela na origem, e terá sela também quando restrito ao subespaço vetorial gerado por $\left\{v_{1}, v_{2}\right\}$.

Logo ,

$\mathrm{P}_{\mathrm{A}}^{\prime}=\mathrm{Q}_{\mathrm{A}}^{\prime}+\mathrm{R}_{\mathrm{A}}^{\prime}$ tem sela na origem 
III-4 PROPRIEDADES DE $\mathrm{f}_{\mathrm{A}}^{\prime}$

A $\in M_{n}(\mathbb{R})$ simétrica, definida positiva.

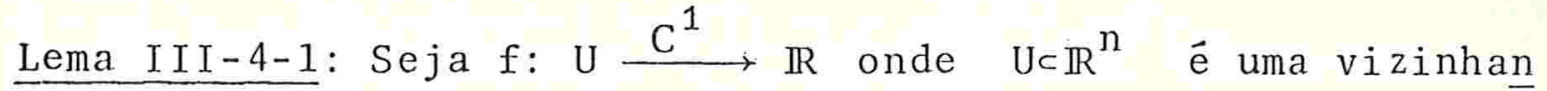
ça aberta de origem e $f(0)=0$.

Se para algum $\varepsilon>0$ tal que $B_{\varepsilon} \subset U$ existe $x_{\varepsilon} \in B_{\varepsilon}^{\prime}$ satisfazendo $f\left(x_{\varepsilon}\right)>0$ (respect. $f\left(x_{\varepsilon}\right)<0$, $f\left(x_{\varepsilon}\right)=0$ ) então existe $\tilde{x}_{\varepsilon} \in B_{\varepsilon}^{\prime}$ tal que $f_{A}^{\prime}\left(\tilde{x}_{\varepsilon}\right)>0$ (respect. $f_{A}^{\prime}\left(\tilde{x}_{\varepsilon}\right)<0, f_{A}^{\prime}\left(\tilde{x}_{\varepsilon}\right)=0$ ).

8 Consideremos a curva $\Gamma \subset \mathbb{R}^{\mathrm{n}}$ cujos extremos são a origem e $x_{\varepsilon}$, e que intercepta ortogonalmente as curvas de níve1 da função

$\mathrm{F}: \mathbb{R}^{\mathrm{n}} \longrightarrow \mathbb{R}$

$x \longmapsto\langle x| A x>$

Seja $x:(-\infty, 0] \longrightarrow \mathbb{R}^{n}$<smiles>[14CH3][14C]([14CH3])=[14C][Te]</smiles>

$x$ é uma parametrização $C^{1}$ de $\Gamma-\{0\}$.

$\Gamma \subset B_{\varepsilon}$, portanto fica bem definida a função

f : $(-\infty, 0] \stackrel{\mathrm{C}^{1}}{\longrightarrow} \mathbb{R}$

$t \longmapsto f \circ x(t)$

Como $\mathfrak{f}(0)=f\left(x_{\varepsilon}\right)$ e $\lim _{t \rightarrow-\infty} f(t): 0$, existe $\bar{t} \in(-\infty, 0)$ tal que $\frac{d f}{d t}(\bar{t})$ tem mesmo sinal que $f\left(x_{\varepsilon}\right)$.

Por outro 1ado, 


$$
\begin{aligned}
& f_{A}^{\prime}(x(\bar{t}))=\langle\operatorname{gradf}(x(\bar{t})) \mid A x(\bar{t})\rangle=\frac{d f}{d t}(\bar{t}) \\
& \text { Logo, para } \tilde{x}_{\varepsilon}=x(\bar{t}) \text { segue a tese }
\end{aligned}
$$

A seguir enunciaremos, para $f_{A}^{\prime}$, proposições anälogas àquelas enunciadas no capítulo II para $f^{\prime}$. Elas decorrem, to das, do Lema III-4-1 (que è uma generalização do Lema II-2-1, e desempenha, aqui, o mesmo papel desempenhado no capítulo II por este).

No que se segue, consideraremos $f:\left(\mathbb{R}^{n} ; 0\right) \stackrel{C^{1}}{\longrightarrow}(\mathbb{R} ; 0)$

Prop.III-4-1: Se $f_{A}^{\prime}$ tem extremo forte na origem, então $f$ tam bém tem extremo forte na origem, e este è do mesmo tipo que o de $f_{A}^{\prime}$.

Prop.III-4-2: Se $f$ tem mínimo forte (respect. mäximo forte) na origem, então a origem é ponto de mínimo forte, mínimo brando ou sela (respect. máximo forte, máximo brando ou sela) para $f_{A}^{\prime}$, e efetí vamente cada uma dessas possibilidades se concretiza.

Prop.III-4-3: Se f tem mínimo brando (respect. máximo brando) na origem, então a origem é ponto de mínimo brando ou sela (respect. máximo brando ou sela) para $f_{A}^{\prime}$, e cada uma dessas possibilidades de fato ocorre. 
Prop.III-4-4: Se $f$ tem sela na origem, então $f_{A}^{\prime}$ tem sela na origem.

III - 5 CLASSIFICAÇÃO DOS PARES $\left(f ; f_{A}^{\prime}\right)$

Os resultados do parägrafo anterior nos permitem clas sificar completamente os pares ( $f, f_{A}^{\prime}$ ) relativamente aos pares de pontos críticos associados, para $A \in M_{n}(\mathbb{R})$ simétrica, definida positiva, e

$f:\left(\mathbb{R}^{\mathrm{n}} ; 0\right) \stackrel{\mathrm{C}^{1}}{\longrightarrow}(\mathbb{R} ; 0)$

com ponto crítico na origem.

Apresentamos tal classificação na tabela seguinte.

Relembramos que, nos exemplos, $\left\{v_{1}, \ldots, v_{n}\right\}$ è uma base ortonormal de $\mathbb{R}^{\mathrm{n}}$ formada por autovetores de A associados a autovalores (positivos) $\lambda_{1}, \ldots, \lambda_{n}$. 


\begin{tabular}{|c|c|c|}
\hline \multicolumn{2}{|c|}{$\begin{array}{l}\text { Ponto critico } \\
\text { na origem }\end{array}$} & \multirow{2}{*}{$\begin{array}{c}\text { Exemp1o }(n \geq 2) \\
x=\left(x_{1}, \ldots, x_{n}\right) \in \mathbb{R}^{n}\end{array}$} \\
\hline de $f$ & de $\mathrm{f}_{\mathrm{A}}^{\prime}$ & \\
\hline $\begin{array}{l}\pi \\
-1 \\
0 \\
\infty\end{array}$ & $\begin{array}{l}\stackrel{\infty}{-1} \\
\infty \\
\omega\end{array}$ & $x_{1}^{2}-x_{2}^{2}$ \\
\hline 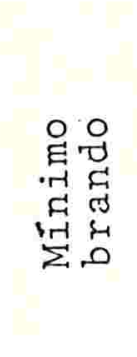 & 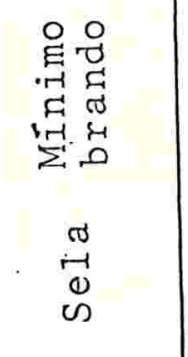 & $\begin{array}{l}\left\langle x \mid v_{1}\right\rangle^{2} \\
\left(\langle x| v_{1}>-\left\langle x \mid v_{2}\right\rangle^{m}\right)^{2} \quad m \in \mathbb{N} \quad, \quad m>2 \frac{\lambda_{1}}{\lambda_{2}}\end{array}$ \\
\hline 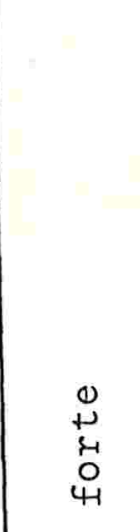 & 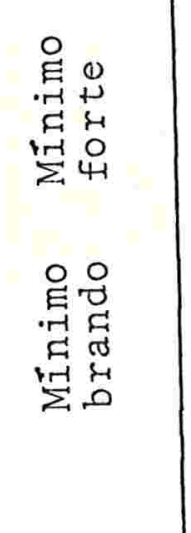 & $\begin{array}{l}\sum_{j=1}^{n}\left\langle x \mid v_{j}\right\rangle^{2} \\
\left(<x\left|v_{1}\right\rangle-\langle x| v_{2}>^{m}\right)^{2}+a<x\left|v_{2}\right\rangle^{2 m}+\sum_{j=3}^{n}<x\left|v_{j}\right\rangle^{2} \\
m \in \mathbb{N}^{*} \quad, \quad m \neq \frac{\lambda_{1}}{\lambda_{2}}, \quad a=\frac{\left(\lambda_{1}-m \lambda_{2}\right)^{2}}{4 m \lambda_{1} \lambda_{2}}\end{array}$ \\
\hline 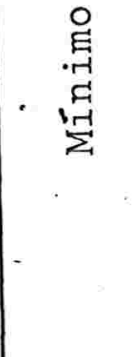 & $\begin{array}{l}\infty \\
-1 \\
\infty \\
\infty\end{array}$ & $\begin{array}{c}\left(\left\langle x \mid v_{1}\right\rangle-\left\langle x \mid v_{2}\right\rangle^{m}\right)^{2}+a<x\left|v_{2}\right\rangle^{2 m}+\sum_{j=3}^{n}\left\langle x \mid v_{j}\right\rangle^{2} \\
m \in \mathbb{N}^{*}, \quad m \neq \frac{\lambda_{1}}{\lambda_{2}}, \quad a<\frac{\left(\lambda_{1}-m \lambda_{2}\right)^{2}}{4 m \lambda_{1} \lambda_{2}}\end{array}$ \\
\hline & e $n$ & ocorrem outras possibilidades \\
\hline
\end{tabular}




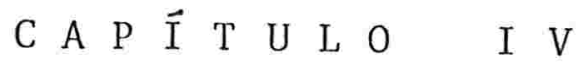

Uma ap1icação à Estabi1idade 
IV-1 INTRODUCÃ̃O

Hā na literatura vārios resultados clâssicos relativos ao estudo da estabilidade de Liapunov de um ponto de equilíbrio de um sistema mecânico conservativo. Em parte desses re sultados utilizam-se funções auxiliares que evidenciam o com portamento particular de todos ou de alguns dos movimentos com condições iniciais próximas do ponto de equilíbrio a ser estudado. Exemplos clássicos disto são o Teorema de Direch-1et-Lagrange (ver [4]) e o Teorema de Chetaev (ver $\$ I V-2)$.

$\mathrm{Na}$ demonstração do Teorema de Direchlet-Lagrange utilí za-se a energia mecânica $H$ como função de Liapunov para estabilidade. Na demonstração do Teorema de Chetaev utiliza-se a função auxiliar $V=-<q \mid p>H$ como função dechetaev.

Estas funções auxiliares são bastante sugestivas: H por ser uma integral primeira, V porque informa que algumas soluções de certos sistemas mecânicos são "arrastadas" para longe da origem.

Já para o Teorema de Liapunov para Instabilidade (ver §IV-2) as demonstrações que têm sido apresentadas não utili zam função de Chetaev.

Nossa pretensão neste capítulo é apresentar uma demons tração de um caso particular desse teorema para sistemas me cânicos com dois graus de liberdade, usando duas funções au xiliares: uma que nos permitirä garantir a invariância de uma certa região no espaço de fase e outra que fará o pape1 
de função "expelente". Tal demonstração assemelhar-se-á muito à do Teorema de Chetaev, já que as duas funções mencionadas desempenharão papel anảlogo ao que a função $V=-\langle\mathrm{g} \mid \mathrm{p}\rangle \mathrm{H}$ de sempenha na demonstração desse terorema.

\section{IV-2 TEOREMA DE LIAPUNOV PARA INSTABILIDADE}

No que segue, $\Lambda \subset \mathbb{R}^{n}$ denotará uma vizinhaça aberta da origem. Como è usual em mecânica, usaremos também as notações:

Energia potencial

$$
\pi: \Lambda \stackrel{\mathrm{C}^{2}}{\longrightarrow} \mathbb{R} \quad, \quad \mathrm{j}^{1} \pi(0)=0
$$

Energia cinëtica

$$
\begin{aligned}
\mathrm{T}: \Lambda \times \mathbb{R}^{\mathrm{n}} \stackrel{\mathrm{C}^{2}}{\longrightarrow} & \mathbb{R} \\
(\mathrm{q}, \mathrm{p}) \longmapsto & \longrightarrow
\end{aligned}
$$

onde $D_{\epsilon} C^{2}\left(\Lambda, M_{n}(\mathbb{R})\right)$ e $D(q)$ é simétrica, definida positiva, $\forall \mathrm{q} \in \Lambda$.

$\underline{\text { Hamil toniano }}$

$$
\begin{aligned}
H: & \Lambda \times \mathbb{R}^{n} \stackrel{\mathrm{C}^{2}}{\longrightarrow} \mathbb{R} \\
(\mathrm{q}, \mathrm{p}) & \longrightarrow \mathrm{H}(\mathrm{q}, \mathrm{p})=\mathrm{T}(\mathrm{q}, \mathrm{p})+\pi(\mathrm{q})
\end{aligned}
$$

Sistema mecânico conservativo de energia potencial $\pi$ e energia cinētica $\mathrm{T}$

(1) $\left\{\begin{array}{l}\dot{q}=\frac{\partial H}{\partial p} \\ \dot{p}=-\frac{\partial H}{\partial q}\end{array}\right.$ 
onde $\frac{\partial H}{\partial q}$ e $\frac{\partial H}{\partial p}$ denotam o gradiente de $H$ com respeito a $q$ e a p respectivamente.

Teorema de Liapunov para Instabilidade:

Se $\pi$ não tem mínimo em $q=0$ e este fato é 2-de cidíve1, então a origem do sistema mecânico (1) é instāve1. $(\operatorname{ver}[4])$

Teorema de Chetaev:

$$
\begin{aligned}
& \text { Se existe } \varepsilon>0 \quad(\operatorname{com} \underset{\varepsilon}{\vec{B} \subset \Lambda)} \text { tal que para } \\
& \theta=\left\{q \in B_{\varepsilon} \mid \pi(q)<0\right\} \text { temos } \\
& \text { (i) } 0 \in \partial \theta \\
& \text { (ii) }<\frac{\partial \pi}{\partial q} \mid q><0 \quad \forall q \in \theta \\
& \text { então a origem do sistema mecânico (1) é instá } \\
& \text { vel. }
\end{aligned}
$$

Observação: Este teorema é um caso particular do teorema que será demonstrado a seguir, com a função natural $V=-<q \mid p>H$ de sempenhando o pape1 de W.

Apesar do próximo resultado ser enunciado para um siste ma de equações diferenciais particular -um sistema mecânico conservativo- é relevante notar que è um resultado geral, com a mesma demonstração, bastando para isto substituir $\mathrm{H}$ no enunciado por uma integral primeira. 
Teor.IV-2-1: Seja $\Omega=\left\{(q, p) \in \Lambda \times \mathbb{R}^{n} \mid H(q, p)<0\right\}$

Se existirem um aberto $\omega \subset \Omega$ não vazio, aderente à origem e uma função

$W: \Lambda \times \mathbb{R}^{n} \longrightarrow \mathbb{R}$

tais que, para algum $\varepsilon>0$ ( $\operatorname{com} \bar{B}_{\varepsilon} q x \times \mathbb{R}^{n}$ )
(i) $W>0$
em
$\omega_{\varepsilon}=\omega \bar{B}_{\varepsilon}$
(ii) $\dot{W}>0$
em
$\Omega_{\varepsilon}=\Omega_{\cap} \overline{\mathrm{B}} \varepsilon$

então a origem do sistema mecânico (1) è instāve1.

8 (a) Para cada $\delta>0$, o fechado

$$
\Omega^{\delta}=\{(\mathrm{q}, \mathrm{p}) \in \Omega \mid \mathrm{H}(\mathrm{q}, \mathrm{p}) \leq-\delta\}
$$

è invariante, pois $\mathrm{H} \overrightarrow{\mathrm{e}}$.constante ao longo das traje tórias do sistema mecânico (1).

(b) $W>0$ em $\omega_{\varepsilon}$.

(c) Se $\Omega_{\varepsilon}^{\delta}=\Omega^{\delta}{ }_{n B}$ for não vazio, temos $\dot{W}>0$ em $\Omega_{\varepsilon}^{\delta}$.

Assim, se um movimento tem condição inicial $\left(q_{0}, p_{0}\right) \in \omega_{\varepsilon}$ tomando-se $\delta=\frac{W\left(q_{0}, p_{0}\right)}{2}>0$ segue de (a), (b) e (c) que ta1 movimento tem que abandonar o compacto $\Omega_{\varepsilon}^{\delta}$ por um ponto de sua fronteira que esteja em $S_{\varepsilon}$

Enunciaremos agora uma versão mais fraca do Teorema de Liapunov para Instabilidade, versão esta que pretendemos demonstrar usando o resultado anterior. 
Teor.IV-2-2: Seja $\Lambda \subset \mathbb{R}^{2}$ vizinhança aberta de $0 \in \mathbb{R}^{2}$.

Se $\pi: \Lambda \stackrel{\mathrm{C}^{2}}{\longrightarrow} \mathbb{R}$ tem sela 2 -decidíve1 em 0 então a origem do sistema mecânico (1) é instāve1.

Por recair nas hipóteses do teorema IV-2-1, desenvolvere mos dois lemas.

Lema IV-2-2a: Seja $f: \mathbb{R}^{2} \stackrel{C^{2}}{\longrightarrow} \mathbb{R}, f(0)=0$.

Se (i) $f$ tem sela 2-decidível na origem.

(ii) $j^{2} f$ se anula exatamente nas retas $y= \pm a x \quad(a>0)$

(iii) $j^{2} f(0, y)<0$ se $y \neq 0$, então existem $A \in M_{2}(\mathbb{R})$ diagonal, tão perto da identidade quanto se queira, e $\varepsilon_{0}>0$ tais que $\left.\begin{array}{l}(x, y) \in B_{\varepsilon_{0}}^{\prime} \\ f(x, y) \leq 0\end{array}\right\} \Rightarrow f_{A}^{\prime}(x, y)<0$

8 Por (ii) e (iii) temos que $p=j^{2} f$ é dado por

$$
\begin{aligned}
P(x, y) & =(a x-y)(a x+y)=a^{2} x^{2}-y^{2} \\
\text { Seja } \quad A & =\left[\begin{array}{cc}
\alpha^{2} & 0 \\
0 & 1
\end{array}\right] \quad, \quad \alpha>0 \\
P_{A}^{\prime}(x, y) & =\left\langle\operatorname{grad} P(x, y) \mid\left(\alpha^{2} x, y\right)\right\rangle \\
& =2\left(a^{2} \alpha^{2} x^{2}-y^{2}\right)
\end{aligned}
$$

Por outro lado, $P_{A}^{\prime}=j^{2} f_{A}^{\prime}$ 
Assim, $f=P+R \quad, \quad f^{\prime}=P_{A}^{\prime}+\tilde{R} \quad, \quad j^{2} R=j^{2} \tilde{R}=0$

Seja $\alpha \in(0,1)$ arbitrário. Fixemos $b \in(a \alpha, a)$.

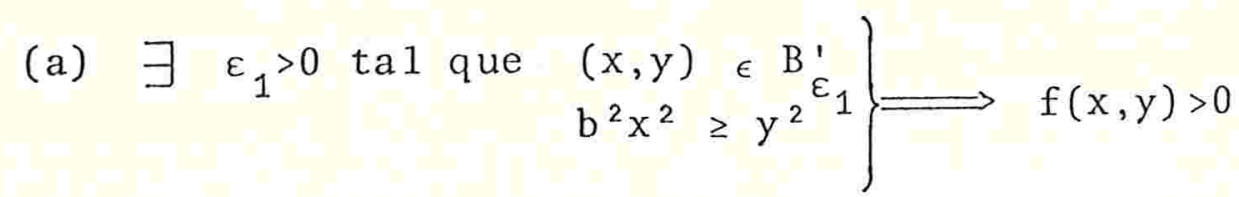

De fato:

$\operatorname{Se}(x, y) \neq 0 \in \mathbb{R}^{2} \quad$ e $\quad b^{2} x^{2} \geq y^{2} \quad$ temos

$\frac{b^{2} x^{2}-y^{2}}{|(x, y)|^{2}} \geq 0 \quad$ e $\quad|(x, y)|^{2} \leq\left(b^{2}+1\right) x^{2}$,

e como $0<\mathrm{b}<\mathrm{a}$ segue que

$$
\begin{aligned}
\frac{f(x, y)}{|(x, y)|^{2}} & =\frac{b^{2} x^{2}-y^{2}}{|(x, y)|^{2}}+\frac{\left(a^{2}-b^{2}\right) x^{2}}{|(x, y)|^{2}}+\frac{R(x, y)}{|(x, y)|^{2}} \geq \\
& \geq \frac{a^{2}-b^{2}}{b^{2}+1}+\frac{R(x, y)}{|(x, y)|^{2}}
\end{aligned}
$$

Logo, tomando-se $\varepsilon_{1}>0$ tal que

$$
\frac{R(x, y)}{|(x, y)|^{2}}<\frac{a^{2}-b^{2}}{2\left(b^{2}+1\right)}, \quad \forall(x, y) \in B_{\varepsilon_{1}}^{\prime}, \quad \text { segue }(a) \text {. }
$$

Com um raciocínio anālogo ao anterior concluimos que

(b) $\exists \varepsilon_{2}>0$ tal que $\left.\begin{array}{c}(x, y) \in B_{\varepsilon_{2}}^{\prime} \\ b^{2} x^{2} \leq y^{2}\end{array}\right\} \Longrightarrow f_{A}^{\prime}(x, y)<0$

Logo, tomando-se $\quad \varepsilon_{0}=\min \left\{\varepsilon_{1}, \varepsilon_{2}\right\}$

temos, para $(x, y) \in B_{\varepsilon_{\dot{o}}^{\prime}}^{\prime}:$

$$
f(x, y) \leq 0 \stackrel{(a)}{\longrightarrow} b^{2} x^{2}-y^{2} \stackrel{(b)}{\longrightarrow} f_{A}^{\prime}(x, y)<0
$$




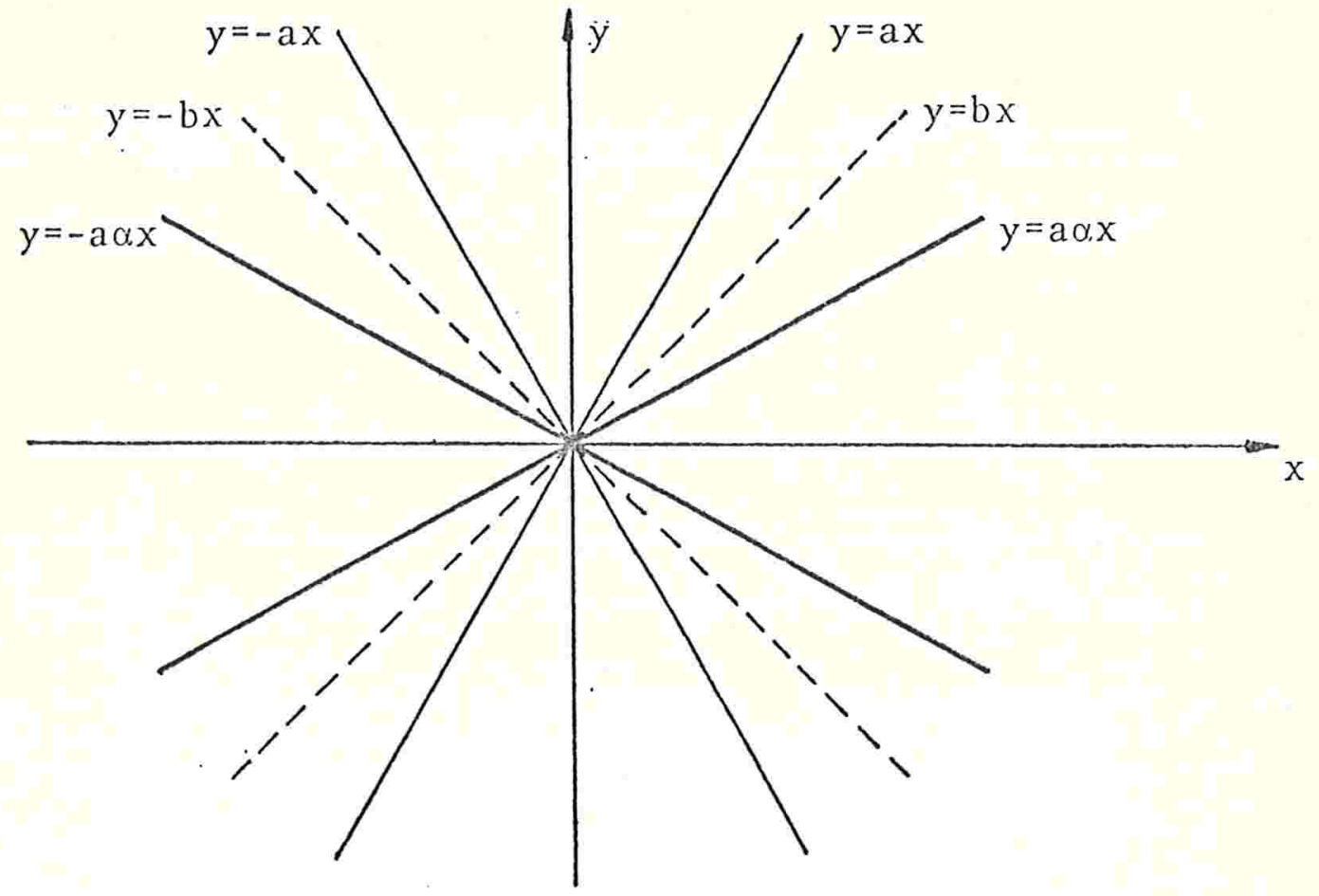

Lema IV-2-2b: Seja $\pi: \Lambda \stackrel{\mathrm{C}^{2}}{\rightarrow} \mathbb{R}\left(\Lambda \subset \mathbb{R}^{2}\right.$ vizinhança aberta de 0) uma energia potencial com sela 2-decidível na origem.

Suponhamos que $\mathrm{j}^{2} \pi$ se anula exatamente nas retas $\mathrm{q}_{2}= \pm \mathrm{aq}_{1}($ para algum a>0) e que $j^{2} \pi\left(0, q_{2}\right)<0$ se $q_{2} \neq 0$.

Seja $\Omega=\left\{(q, p) \in \Lambda x \mathbb{R}^{2} \mid H(q, p)<0\right\}$

Então existem $A \in M_{2}(\mathbb{R})$ definida positiva, um aberto não vazio $\omega \subset \Omega$ aderente à origem e $\varepsilon>0$. $\left(\operatorname{com} \overline{\mathrm{B}}_{\varepsilon} \subset \Lambda \times \mathbb{R}^{2}\right)$ tais que a função $\mathrm{W}: \Lambda \times \mathbb{R}^{2} \stackrel{\mathrm{C}^{1}}{\longrightarrow} \mathbb{R}$

$$
\text { satisfaz } \begin{cases}(\mathrm{q}, \mathrm{p}) \longmapsto & \longmapsto W(q, p)=-<q \mid A p>H \\ (i) \quad W>0 & \text { em } \dot{\omega}_{\varepsilon}=\omega \cap \bar{B}_{\varepsilon}\end{cases}
$$


8 Pelo 1ema IV-2-2a, existem $\mathrm{A}=\left[\begin{array}{ll}\bar{\alpha} & \overline{0} \\ 0 & \bar{\beta}\end{array}\right]$ definida positiva tão perto da identidade quanto se queira e $\tilde{\varepsilon}_{0}>0$ (podemos supor que $B_{\tilde{\varepsilon}_{0}} \subset \Lambda$ ) tais que

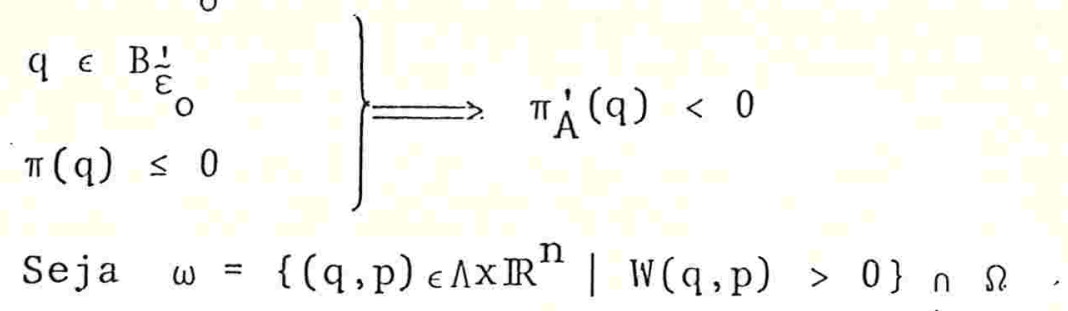

(a) $\omega \vec{e}$ aberto, não vazio, aderente à origem.

De.fato, arbitrariamente perto da origem de $\mathbb{R}^{2}$ existe $\bar{q} \neq 0$ tal que $\pi(\bar{q})<0$. Para $p \in \mathbb{R}^{2}$ tal que $|p| \bar{e}$ suficientemente pequena, $H(\bar{q}, p)<0$. Em particular, se $\lambda>0$ é suficientemente pequeno e $\bar{p}=\lambda q$ temos $(\bar{q}, \bar{p}) \in \omega$.

(b) Para uma escolha conveniente de A, existe $\varepsilon>0$ tal que $W$ satisfaz (i) e (ii).

$\psi: \quad \Lambda \times \mathbb{R}^{2} \times \mathbb{R}^{2}-\mathbb{R}$

definida por

$\psi(q, p, \alpha, \beta)=2\left\langle p \mid B(q)\left[\begin{array}{ll}\alpha & 0 \\ 0 & \beta\end{array}\right] p\right\rangle-\left\langle q \mid\left[\begin{array}{ll}\alpha & 0 \\ 0 & \beta\end{array}\right] \frac{\partial \mathrm{T}}{\partial q}\right\rangle$

$\psi$ é continua e para cada $\overline{\mathrm{p}} \epsilon \mathrm{S}^{1}, \psi(0, \overline{\mathrm{p}}, 1,1)>0$

Da compacidade de $S^{1}$ e da continuidade de $\psi$ segue que existe $\delta>0\left(\operatorname{com} B_{\delta} \subset \Lambda\right)$ tal que se

$$
|q|<\delta,|\alpha-1|<\delta, \quad|\beta-1|<\delta \text { e } p \in S^{1}
$$

então $\quad \psi(q, p, \alpha, \beta)>0$ 
Fixemos $A=\left[\begin{array}{ll}\tilde{\alpha} & 0 \\ 0 & \tilde{\beta}\end{array}\right] \in M_{2}(\mathbb{R}) \quad$ com a restrição suplemen$\operatorname{tar}|\tilde{\alpha}-1|<\delta$ e $|\tilde{\beta}-1|<\delta$ e consideremos o $\tilde{\varepsilon}_{0}$ correspondente.

Temos então

$$
\begin{aligned}
& \psi(\mathrm{q}, \mathrm{p}, \tilde{\alpha}, \tilde{\beta})>0 \text { em } \quad \mathrm{B}_{\delta} \times \mathrm{S}^{1} \subset \Lambda \times \mathbb{R}^{2} \\
& \text { Como } \psi(\mathrm{q}, \mathrm{t} p, \tilde{\alpha}, \tilde{\beta})=t^{2} \psi(\mathrm{q}, \mathrm{p}, \tilde{\alpha}, \tilde{\beta}) \quad \forall \quad t \in \mathbb{R}
\end{aligned}
$$

segue que

$$
\begin{array}{lc}
\psi(q, p, \tilde{\alpha}, \tilde{\beta},)>0 \quad \forall \quad q_{\delta}, \quad \forall p \neq 0 \\
\text { Sejam } \varepsilon<\min \left\{\delta, \tilde{\varepsilon}_{0}\right\} & (\varepsilon>0), \\
\Omega_{\varepsilon}=\Omega \cap \bar{B}_{\varepsilon} \text { e } & \omega_{\varepsilon}=\omega \cap \bar{B}_{\varepsilon} . \\
\text { (i) } W(q, p)>0 & \forall(q, p) \in \omega_{\varepsilon},
\end{array}
$$

pois $\mathrm{H}<0$ em $\Omega,<\mathrm{q} \mid \mathrm{Ap}>>0$ em $\omega$ e $\omega_{\varepsilon} \subset \Omega \cap \omega$

(ii) $\dot{W}(q, p)>0 \quad \forall(q, p) \in \Omega_{\varepsilon}$ com $p \neq 0$

De fato

$$
\begin{aligned}
\dot{W}(q, p) & =-H(\langle\dot{q} \mid A p\rangle+\langle q \mid A \dot{p}\rangle) \\
& =-H\left(\left\langle\frac{\partial H}{\partial p} \mid A p\right\rangle-\left\langle q \mid A \frac{\partial H}{\partial q}\right\rangle\right) \\
& =-H\left(2\left\langle p \mid B(q) A p^{\prime}\right\rangle-\left\langle q \mid A \frac{\partial T}{\partial q}\right\rangle-\left\langle q \mid A \frac{\partial \pi}{\partial q}\right\rangle\right) \\
& =-H\left(\psi(q, p, \tilde{\alpha}, \tilde{\beta})-\pi_{A}^{\prime}(q)\right)
\end{aligned}
$$

Agora, como $\forall(q, p) \in \Omega_{\varepsilon}$ temos $\pi(q) \leq H(q, p)<0$ segue que $\pi_{A}^{\prime}(q)<0, \forall(q, p) \in \Omega_{\varepsilon}$. Alëm disso, $\Psi(\mathrm{q}, \mathrm{p}, \tilde{\alpha}, \tilde{\beta}) \geq 0 \quad \forall(\mathrm{q}, \mathrm{p}) \in \Omega_{\varepsilon}$.

Logo, $\dot{W}>0$ em $\Omega_{\varepsilon}$ 
Prova do Teorema IV-2-2:

8 Se $\pi: \Lambda \frac{C^{2}}{R}$ tem sela 2-decidivel na origem $\left(\Lambda \subset \mathbb{R}^{2}\right)$, fazendo-se uma escolha conveniente do sistema de coordenadas de $\mathbb{R}^{2}$, podemos supor que $j^{2} \pi$ se anula exatamente sobre as retas $q_{2}= \pm a q_{1}$ e que $j^{2} \pi\left(0, q_{2}\right)<0$ se $q_{2} \neq 0 \quad(a>0)$.

A instabilidade da origem decorre imediatamente do Teorema IV-2-1 usando-se o Lema IV-2-2b.

\section{IV-3 COMENTARIOS}

Não pudemos apresentar uma demonstração "tipo Chetaev" para o Teorema IV-2-2 no caso de n graus de liberdade para $n \geq 3$, seguindo a linha da apresentação feita quando $n=2$. Isso não foi decorrência de dificuldades técnicas menos elementa res; encontramos, sim, uma barreira intransponíve1: o resul tado do Lema IV-2-2a, que é o suporte da nossa demonstração, não se extende para dimensão $n \geq 3$.

o exemp1o seguinte justificarâ a afirmação que acaba-mos de fazer:

Exemp1o IV-3-1:

$$
\begin{aligned}
& \text { Seja } f: \mathbb{R}^{3}-\mathbb{R} \\
& f(x, y, z)=x^{2}-y^{2}+z^{4} . \\
& f \text { tem sela 2-decidive1 na origem. }
\end{aligned}
$$


Para $A=\left[\begin{array}{lll}\alpha & \beta & \gamma \\ \beta & \delta & \sigma \\ \gamma & \sigma & \eta\end{array}\right] \in M_{3}(\mathbb{R})$ definida positiva

temos

$f_{A}^{\prime}=2 x(\alpha x+\beta y+\gamma z)-2 y(\beta x+\delta y+\sigma z)+4 z^{3}(\gamma x+\sigma y+\eta z)$

Portanto

$f\left(0, z^{2}, z\right)=0 \quad e$

$f_{A}^{\prime}\left(0, z^{2}, z\right)=2 \delta z^{4}-2 \sigma z^{3}+4 \sigma z^{5}+4 \eta z^{4}$

$=-2 \sigma z^{3}+2(2 \eta-\delta) z^{4}+4 \sigma z^{5}$

Para termos a implicação

$$
\begin{aligned}
& " f(x, y, z) \leq 0 \Longrightarrow f_{A}^{\prime}(x, y, z)<0 " \\
& 0 \ll|(x, y ; z)|<\delta
\end{aligned}
$$

seria necessario
a) $\sigma=0$
e
b) $2 n-\delta=0$

Mas a condição b) implicaria em não podermos escolher A suficientemente próxima da identidade. 
BIBLI OGRAFIA:

1 - ANDREOTTI, Aldo \& FRANKEL, Theodore. 1959. The 1efschetz theorem on hyperplane sections. Annals of Mathematics, $69(3): 713-7$.

2 - ATIYAH, M.F. \& MACDONALD, I.G. 1969. Introduction to commutative algebra. London, Addison-Wesley. $128 \mathrm{p}$.

3 - BARONE NETTO, Ange1o. 1980. Extremos detectáveis por jatos: São Paulo, 21p. Tese (Livre-Docência) IME-USP.

4 - LALOY, M.; HABBETS, P. \& ROUCHE, N. 1977. Stability theory by Liapunov's Direct Method. New York, Springer Verlag. 395p. (Spplied Mathematical Sciences, 22).

5 - MILNOR, John. 1968. Singular points of complex hipersurfaces. Princenton, University Press. 122p. (Anna1s of Mathematics Studies, 61).

6 - WHITNEY, Hassler. 1957. Elementary structure of real a1gebraic varieties. Annals of Mathematics, 66(3): 545-54. 